\title{
Fine-Tuning the Odds in Bayesian Networks *
}

\author{
Bahare Salmani ${ }^{1}[0000-0003-3571-2502]$ \\ and Joost-Pieter Katoen ${ }^{10000-0002-6143-1926]}$ \\ RWTH Aachen University, Aachen, Germany \\ \{salmani, katoen\}@cs.rwth-aachen.de
}

\begin{abstract}
This paper proposes new analysis techniques for Bayes networks in which conditional probability tables (CPTs) may contain symbolic variables. The key idea is to exploit scalable and powerful techniques for synthesis problems in parametric Markov chains. Our techniques are applicable to arbitrarily many, possibly dependent, parameters that may occur in multiple CPTs. This lifts the severe restrictions on parameters, e.g., by restricting the number of parametrized CPTs to one or two, or by avoiding parameter dependencies between several CPTs, in existing works for parametric Bayes networks (pBNs). We describe how our techniques can be used for various $\mathrm{pBN}$ synthesis problems studied in the literature such as computing sensitivity functions (and values), simple and difference parameter tuning, ratio parameter tuning, and minimal change tuning. Experiments on several benchmarks show that our prototypical tool built on top of the probabilistic model checker Storm can handle several hundreds of parameters.
\end{abstract}

\section{Introduction}

Parametric Bayesian networks. We consider Bayesian networks (BNs) whose conditional probability tables (CPTs) contain symbolic parameters such as $x_{1}$, $2 \cdot x_{1}^{2}$, and $x_{1}+x_{2}$ with $0<x_{1}, x_{2}<1$. Parametric probabilistic graphical models received a lot of attention, see e.g., [18|19|26|34|40|9|10|11|37|13|14|17|42|7. Sensitivity analysis determines the effect of the parameter values in the CPTs on the decisions drawn from the parametric $\mathrm{BN}$ (pBN), e.g., whether $\operatorname{Pr}(H=h \mid$ $E=e)>q$ for a given $q \in \mathbb{Q} \cap[0,1]$. It amounts to establishing a function expressing an output probability in terms of the $x_{i}$ parameters under study. Parameter synthesis on pBNs deals with instantiating or altering the parameters such that the resulting BN satisfies some constraint of interest. For pBNs, synthesis mostly amounts to parameter tuning: find the minimal change on the parameters such that some constraint, e.g., $\operatorname{Pr}(H=h \mid E=e)>q$ holds [15]40. As sensitivity

* This is a pre-copyedited version of a contribution published in Vejnarová J., Wilson N. (eds) Symbolic and Quantitative Approaches to Reasoning with Uncertainty. ECSQARU 2021. Lecture Notes in Computer Science, vol 12897. Springer, Cham, 268-283. The definitive authenticated version is available online via https://doi. org/10.1007/978-3-030-86772-0_20 This work is funded by the ERC AdG Projekt FRAPPANT (Grant Nr. 787914). 
analysis and parameter synthesis are computationally hard in general [40]37/38], many techniques restrict the number of parameters per CPT ( $n$-way for small $n$ [13/17/29]), permit parameter dependencies in several CPTs (single CPT [14]), or consider specific structures such as join trees [37] and require all parameters to occur in the same clique of the junction tree.

Parametric Markov chains. Quite independently, analysis techniques for Markov chains (MCs) have been developed in the formal verification community in the last two decades [23/3931|24|41|21/30|27]; for a recent overview see [35. Parametric MCs (pMCs) are MCs in which transitions are labelled with multivariate polynomials over a fixed set of parameters. Substitution of these variables by concrete values induces a probability distribution over the state space of the MC. Whereas early works focused on computing a rational function over the parameters expressing the reachability probability of a given target state, in the last years significant progress has been made to check whether there exists a parameter valuation inducing a $\mathrm{MC}$ that satisfies a given objective, or to partition the parameter space - the space of all possible parameter values - into "good" and "bad" w.r.t. a given objective, e.g., is the probability to reach some states below (or above) a given threshold $q$ ? The complexity of various pMC synthesis problems is studied in [1/36].

This paper aims to extend the spectrum of parameter synthesis techniques for parametric BNs, i.e., BNs in which arbitrary many CPTs may contain symbolic probabilities, with state-of-the-art and recently developed techniques for parametric MCs. Consider the BN adapted from [22] depicted below. The probability

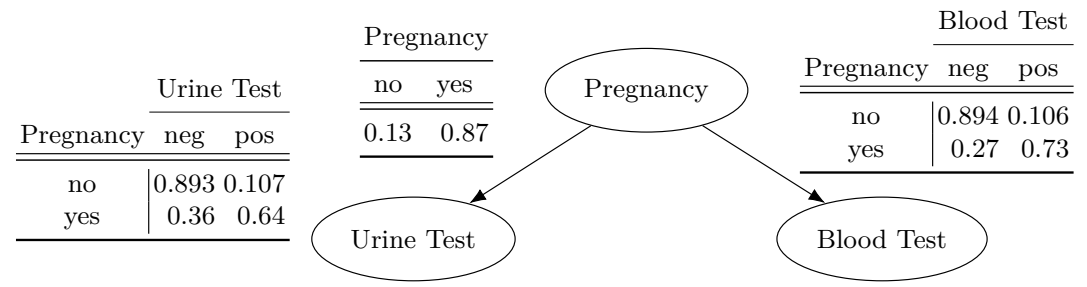

of a cow being pregnant given both tests are negative is about 0.45. Assume the farmer wants to replace both tests such that this false-positive error is below 0.2. Fig. 1 (left) indicates the corresponding $\mathrm{pBN}$ while (right) shows the synthesized values of the parameters $p$ and $q$ (the false-negative probabilities for the new urine and blood tests) using pMC techniques [41] such that the farmer's constraint is satisfied (green) or not (red).

Let us highlight a few issues: we can treat parameter space partitionings that go beyond rectangular shapes, and we support multiple, possibly dependent, parameters (not illustrated in our example). Thanks to approximate techniques such as parameter lifting [41], the entire parameter space can be split into safe, unsafe, and - by some approximation factor - unknown regions. This provides useful information: if it is not possible to find urine and blood tests of a certain quality, are alternatives fine too? Parameter tuning [13] for pBNs finds parame- 


\begin{tabular}{|c|c|c|c|c|}
\hline \multirow[b]{2}{*}{$\mathrm{P}$} & \multicolumn{2}{|c|}{$\mathrm{U}$} & \multirow[b]{2}{*}{$\mathrm{P}$} & B \\
\hline & neg & pos & & neg pos \\
\hline no & 0.893 & 0.107 & no & $\begin{array}{lll}0.894 & 0.106\end{array}$ \\
\hline yes & $\mathrm{p}$ & $1-p$ & yes & $1-\mathrm{q}$ \\
\hline
\end{tabular}

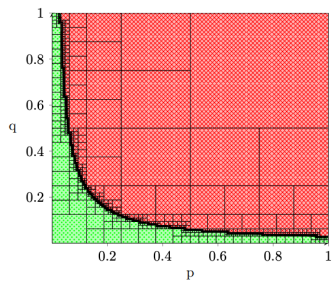

Fig. 1: (left) Parametric CPTs and (right) the parameter space split into safe and unsafe regions for the constraint $\operatorname{Pr}(\mathrm{P}=$ yes $\mid \mathrm{U}=$ neg and $\mathrm{B}=$ neg $) \leq 0.2$.

ter values that are at a minimal distance to the original values in the BN. For our example, the BN sensitivity tool SamIam suggests changing the false-negative probability of the urine test $(p)$ from 0.36 to 0.110456 ; or changing the falsenegative probability of the blood test $(q)$ from 0.27 to $0.0828421^{1}$ Interestingly, if the parameters occur in multiple CPTs, the constraint can be satisfied with a smaller deviation from the original parameter values. Other recent work [5] focuses on obtaining symbolic functions for $\mathrm{pBN}$-related problems such as sensitivity analysis. Note that pBNs are similar to constrained BNs [6 that focus on logical semantics rather than synthesis algorithms and tools as we do.

We treat the theoretical foundations of exploiting pMC techniques for various synthesis questions on pBNs, present a prototypical tool that is built on top of the probabilistic model checker Storm [25] and the pMC analysis tool Prophesy [24], and provide experimental results that reveal:

- pMC techniques are competitive to most common functions with the pBN tools SamIam and Bayesserver ${ }^{2}$

- pMC techniques are well-applicable to general pBNs, in particular by allowing parameter dependencies.

- Approximate parameter space partitioning is effective for parameter tuning e.g., ratio, difference, and minimal change problems.

Further proofs and details of this paper can be found in [44].

\section{Parametric Bayesian networks}

This section defines parametric Bayesian networks $(\mathrm{BNs})^{3}$ and defines the sensitivity analysis and parameter tuning tasks from the literature that we consider. Parameters. Let $X=\left\{x_{1}, \ldots, x_{n}\right\}$ be a finite set of real-valued parameters and $\mathbb{Q}(X)$ denote the set of multivariate polynomials over $X$ with rational coefficients. A parameter instantiation is a function $u: X \rightarrow \mathbb{R}$. A polynomial $f$ can

\footnotetext{
${ }^{1}$ When it comes to multiple parameter suggestions, SamIam suggests $p=0.120097$ or $q=0.089892$.

${ }^{2}$ We do not consider tools such as [48] for sensitivity analysis of Credal networks.

${ }^{3}$ Our notion of parametric BN should not be confused with existing parametric notions that consider variations of the structure, i.e., the topology of the BN.
} 
be interpreted as a function $f: \mathbb{R}^{n} \rightarrow \mathbb{R}$ where $f(u)$ is obtained by substitution, i.e., in $f(u)$ each occurrence of $x_{i}$ in $f$ is replaced by $u\left(x_{i}\right)$. To make clear where substitution occurs, we write $f[u]$ instead of $f(u)$ from now on. We assume that all parameters are bounded, i.e., $l b_{i} \leq u\left(x_{i}\right) \leq u b_{i}$ for each parameter $x_{i}$. The parameter space of $X$ is the set of all possible parameter values, the hyper-rectangle spanned by the intervals $\left[l b_{i}, u b_{i}\right]$. A subset $R$ of the parameter space is called a region.

Variables. Let $V=\left\{v_{1}, \ldots, v_{m}\right\}$ be a set of variables. Let $D_{v_{i}}$ denote the domain of variable $v_{i}$. A joint variable valuation is a function $\eta: V \rightarrow D_{v_{1}} \times \ldots D_{v_{m}}$ that maps each variable $v_{i}$ to some $d \in D_{v_{i}}$. Let $\operatorname{Eval}(V)$ denote the set of all joint variable valuations for $V$. For the set $D$, let $\operatorname{Distr}(D)$ denote the set of parametric probability distributions over $D$.

Parametric Bayes networks. A parametric $\mathrm{BN}$ is a $\mathrm{BN}$ in which entries in the conditional probability tables (CPTs) are polynomials over the parameters in $X$.

Definition 1. A parametric $B N(p B N) \mathcal{B}$ is a tuple $(V, W, X, \Theta)$ with:

$-V=\left\{v_{1}, \ldots, v_{m}\right\}$ is a set of discrete random variables with $\operatorname{dom}\left(v_{i}\right)=D_{v_{i}}$

- $G=(V, W)$ with $W \subseteq V \times V$ is a directed acyclic graph on $V$

$-X=\left\{x_{1}, \ldots, x_{n}\right\}$ is a finite set of real-valued parameters

- $\Theta$ is a set of parametric conditional probability tables $\Theta=\left\{\Theta_{v_{i}} \mid v_{i} \in V\right\}$. Let parents $\left(v_{i}\right)$ denote the set of parents for the node $v_{i}$ in $G$. The CPT for variable $v_{i}$ is the function $\Theta_{v_{i}}: \operatorname{Eval}\left(\right.$ parents $\left.\left(v_{i}\right)\right) \rightarrow \operatorname{Distr}\left(D_{v_{i}}\right)$ that maps each evaluation $\overline{p a r} \in \operatorname{Eval}\left(\right.$ parents $\left.\left(v_{i}\right)\right)$ to a parametric probability distribution $\Theta_{v_{i}}(\overline{p a r})$ over $D_{v_{i}}$. The CPT entry $\Theta_{v_{i}}(\overline{p a r})\left(d_{i}\right)$ denotes the probability of $v_{i}=d_{i}$ given the parents evaluation $\overline{p a r}$.

Let $\mathcal{B}[u]$ be obtained by replacing every parameter $x_{i}$ in $\mathcal{B}$ by its value $u\left(x_{i}\right)$. A parameter instantiation $u$ is well-formed for the $\mathrm{pBN} \mathcal{B}$ if $\mathcal{B}[u]$ is a $\mathrm{BN}$, i.e., for every $v_{i} \in V$ and parent evaluation $\overline{p a r}, \Theta_{v_{i}}(\overline{p a r})[u]$ yields a probability distribution over $D_{v_{i}}$. In the sequel, we assume $u$ to be well-formed. A pBN defines a parametric joint probability distribution over $V$.

$p B N$ subclasses. We define some sub-classes of $\mathrm{pBNs}$ that are used in existing sensitivity analysis techniques and tools. They constrain the number of parameters, the number of CPTs (and the number of rows in a CPT) containing parameters. Let $\mathcal{B}=(V, W, X, \Theta)$ be a pBN, $c\left(x_{i}\right)$ the number of CPTs in $\mathcal{B}$ in which $x_{i}$ occurs and $r\left(x_{i}\right)$ the number of CPT rows in which $x_{i}$ occurs.

$-\mathcal{B} \in p_{1} c_{1} r_{1}$ iff $\mathcal{B}$ contains one parameter $x_{1}$ and $x_{1}$ only occurs in a single row of a single CPT, i.e., $X=\left\{x_{1}\right\}, c\left(x_{1}\right)=r\left(x_{1}\right)=1$.

$-\mathcal{B} \in p_{2} c_{\leq 2} r_{1}$ iff $\mathcal{B}$ involves two parameters occurring only in two rows of two (or one) CPTs, i.e., $X=\left\{x_{1}, x_{2}\right\}, c\left(x_{i}\right) \in\{1,2\}, r\left(x_{i}\right)=1$ for $i=1,2$.

- $\mathcal{B} \in p_{*} c_{1} r_{1}$ iff $\mathcal{B}$ allows multiple distinct parameters, provided each parameter occurs in a single row of a single CPT, i.e., $r\left(x_{i}\right)=1, c\left(x_{i}\right)=1$ for each $x_{i}$ and all the parameters occur in the same CPT. 
The class $p_{1} c_{1} r_{1}$ is used in one-way, $p_{2} c_{\leq 2} r_{1}$ in two-way sensitivity analysis [13]17|29] and $p_{*} c_{1} r_{1}$ in single CPT [14].

Parameter synthesis problems in $p B N$. We define some synthesis problems for pBNs by their corresponding decision problems [38. Let Pr denote the parametric joint distribution function induced by $\mathrm{pBN} \mathcal{B}=(V, W, X, \Theta)$ and $\operatorname{Pr}[u]$ the joint probability distribution of $\mathcal{B}[u]$ at well-formed instantiation $u$. Let $E \subseteq V$ be the evidence, $H \subseteq V$ the hypothesis and $q \in \mathbb{Q} \cap[0,1]$ a threshold.

Parameter tuning. Find an instantiation $u$ s.t. $\operatorname{Pr}[u](H=h \mid E=e) \geq q$. Hypothesis ratio parameter tuning. Find an instantiation $u$ s.t.

$$
\frac{\operatorname{Pr}[u]\left(H=h^{\prime} \mid E=e\right)}{\operatorname{Pr}[u](H=h \mid E=e)} \geq q \quad \text { i.e., } \quad \frac{\operatorname{Pr}[u]\left(H=h^{\prime}, E=e\right)}{\operatorname{Pr}[u](H=h, E=e)} \geq q,
$$

where $h$ and $h^{\prime}$ are joint variable evaluations for the hypothesis $H$.

Hypothesis difference parameter tuning. Find an instantiation $u$ s.t.

$$
\operatorname{Pr}[u](H=h \mid E=e)-\operatorname{Pr}[u]\left(H=h^{\prime} \mid E=e\right) \geq q,
$$

where $h$ and $h^{\prime}$ are joint variable evaluations for the hypothesis $H$.

Minimal change parameter tuning. For a given parameter instantiation $u_{0}$ and $\epsilon \in \mathbb{Q}_{>0}$, find an instantiation $u$ s.t.

$$
d\left(\operatorname{Pr}[u], \operatorname{Pr}\left[u_{0}\right]\right) \leq \epsilon,
$$

where $d$ is a distance notion on probability distributions, see [15].

Computing sensitivity function and sensitivity value. For the evidence $E=e$ and the hypothesis $H=h$, compute the sensitivity function:

$$
f_{\operatorname{Pr}(H=h \mid E=e)}=\operatorname{Pr}(H=h \mid E=e) .
$$

This is a rational function over $X$, i.e., a fraction $g / h$ with $g, h \in \mathbb{Q}(X)$.

The difference and ratio problems can analogously be defined for evidences. The evidence tuning problems are defined for values $e$ and $e^{\prime}$ for $E$, given a fixed value $h$ for $H$.

\section{Parametric Markov chains}

A parametric Markov chain is a Markov chain in which the transitions are labelled with polynomials over the set $X$ of parameters. These polynomials are intended to describe a parametric probability distribution over the pMC states.

Definition 2. A parametric Markov chain $(p M C) \mathcal{M}$ is a tuple $\left(S, s_{0}, X, P\right)$ where $S$ is a finite set of states with initial state $s_{0} \in S, X$ is as before, and $P: S \times S \rightarrow \mathbb{Q}(X)$ is the transition probability function. 
For pMC $\mathcal{M}=\left(S, s_{0}, X, P\right)$ and well-formed parameter instantiation $u$ on $X$, $\mathcal{M}[u]$ is the discrete-time Markov chain $\left(S, s_{0}, X, P[u]\right)$ where $P[u]$ is a probability distribution over $S$. We only consider well-formed parameter instantiations.

Reachability probabilities. Let $\mathcal{D}$ be an MC. Let $\operatorname{Paths}(s)$ denote the set of all infinite paths in $\mathcal{D}$ starting from $s$, i.e., infinite sequences of the form $s_{0} s_{1} s_{2} \ldots$ with $P\left(s_{i}, s_{i+1}\right)>0$. A probability measure $\operatorname{Pr}_{\mathcal{D}}$ is defined on measurable sets of infinite paths using a standard cylinder construction; for details, see, e.g., [2, Ch. 10]. For $G \subseteq S$, let

$$
\underset{\mathcal{D}}{\operatorname{Pr}}(\diamond G)=\operatorname{Pr}_{\mathcal{D}}\left\{s_{0} s_{1} s_{2} \ldots \in \operatorname{Paths}\left(s_{0}\right) \mid \exists i . s_{i} \in G\right\}
$$

denote the probability to eventually reach some state in $G$ from $s_{0}$. For pMC $\mathcal{M}$, $\operatorname{Pr}_{\mathcal{M}}(\diamond G)$ is a function with $\operatorname{Pr}_{\mathcal{M}}(\diamond G)[u]=\operatorname{Pr}_{\mathcal{D}}(\diamond G)$ where $\mathcal{D}=\mathcal{M}[u]$, see [23].

Parameter synthesis problems on pMCs. We consider the following synthesis problems on pMCs. Let $\mathcal{M}=\left(S, s_{0}, X, P\right)$ be a pMC and $\lambda \in \mathbb{Q} \cap[0,1]$ a threshold, $\sim$ a binary comparison operator, e.g., $<$ or $\geq$, and $G \subseteq S$.

Feasibility problem. Find a parameter instantiation $u$ s.t. $\operatorname{Pr}_{\mathcal{M}[u]}(\diamond G) \sim \lambda$. Synthesis problem. Partition a region $R$ into $R_{a}$ and $R_{r}$ s.t.

$$
\operatorname{Pr}_{\mathcal{M}[u]}(\diamond G) \sim \lambda \text { for all } u \in R_{a} \text { and } \underset{\mathcal{M}[u]}{\operatorname{Pr}}(\diamond G) \not \lambda \text { for all } u \in R_{r} .
$$

$R_{a}$ is called an accepting region and $R_{r}$ a rejecting region.

Approximate synthesis problem. Partition a region $R$ into an accepting region $R_{a}$, rejecting region $R_{r}$, and unknown region $R_{u}$, such that $R_{a} \cup R_{r}$ covers at least $c \%$ of $R$. Additionally, $R_{a}, R_{r}$, and $R_{u}$ should be finite unions of rectangular regions.

Verification problem. Check whether region $R$ is accepting, rejecting, or inconsistent, i.e., neither accepting nor rejecting.

Computing reachability functions. Compute the rational function $\operatorname{Pr}_{\mathcal{M}}(\diamond G)$.

Algorithms for pMC synthesis problems. Several approaches have been developed to compute the reachability function $\operatorname{Pr}_{\mathcal{M}}(\diamond G)$. This includes state elimination [23], fraction-free Gaussian elimination [1] and decomposition [33/28]. The reachability function can grow exponentially in the number of parameters, even for acyclic pMCs [1]. Feasibility is a computationally hard problem: finding parameter values for a pMC that satisfy a reachability objective is ETRcomplete [36. Feasibility has been tackled using sampling search methods such as PSO ${ }^{5}$ and Markov Chain Monte Carlo [16] and solving a non-linear optimization problem [4]. State-of-the-art approaches 2021] iteratively simplify the NLP ${ }^{6}$ encoding around a point to guide the search. The approximate synthesis

\footnotetext{
${ }^{4}$ Existential Theory of the Reals. ETR problems are between NP and PSPACE, and ETR-hard problems are as hard as finding the roots of a multi-variate polynomial.

5 Particle swarm optimization.

${ }^{6}$ Nonlinear programming.
} 
problem checking is best tackled with parameter lifting [41]. The parameter lifting algorithm (PLA) first drops all dependencies between parameters in a pMC. It then transforms the pMC into a Markov decision process to get upper and lower bounds for the given objective.

\section{Analysing parametric BNs using pMC techniques}

The key of our approach to tackle various synthesis problems on pBNs is to exploit pMC techniques. To that end, we transform a $\mathrm{pBN}$ into a $\mathrm{pMC}$. We first present a recipe that is applicable to all inference queries on pBNs, and then detail a transformation that is tailored to the evidence in an inference query.

A pBN2pMC transformation. This is inspired by our mapping of BNs onto treeshaped MCs 43. Here, we propose an alternative transformation that yields more succinct (p)MCs, as it only keeps track of a subset of (p)BN variables at each "level" of the (p)MC. Intuitively, the value of variable $v_{i}$ is still remembered if it has already been processed and at least one of its children has not.

Notations. Let $\varrho=\left(v_{1}, \ldots, v_{m}\right)$ be a topological order over the variables in $V$ with respect to the DAG $G$ and $\varrho(v)$ denote the index of variable $v$ in the order. Let $E=E_{1} \wedge \ldots E_{k}$ be the evidence, where $E_{i}$ is in the form of $v_{E_{i}}=d_{E_{i}}$ with $v_{E_{i}} \in V$ and

$$
v_{E_{1}}<\varrho v_{E_{2}}<\varrho \ldots<\varrho v_{E_{k}} \quad \text { i.e., } \quad \varrho\left(v_{E_{1}}\right)<\varrho\left(v_{E_{2}}\right)<\ldots<\varrho\left(v_{E_{k}}\right) .
$$

In the sequel, we often denote $E_{k}$ as $E_{\text {last }}$.

The hypothesis $H$ can be in the form any arbitrary logical formula over the atomic propositions $v_{i}=d_{i}$. W.l.o.g, let $H=H_{1} \wedge \ldots \wedge H_{l}$ be the hypothesis, where $H_{i}$ is in the form of $v_{H_{i}}=d_{H_{i}}$ and $\varrho\left(v_{H_{i}}\right)<\varrho\left(v_{H_{i+1}}\right)$. For the logical formula $A$, let $\operatorname{vars}(A)$ denote the set of variables that appear in $A$. Let $*$ denote the don't care value. For the variable $v_{i} \in V$, let $D_{v_{i}}^{*}=D_{v_{i}} \cup\{*\}$. A variables state is a function $s: D_{v_{1}}^{*} \times \ldots \times D_{v_{m}}^{*}$ that maps each variable $v_{i} \in V$ either to some $d_{i}$ or to the don't care value. Intuitively speaking, $v_{i}=*$ if the value of variable $v \in V$ is either not yet determined or not needed any more. Let $\operatorname{States}(V)$ denote the set of all variables states. Let $s \in \operatorname{States}(V)$.

- We write $s \models\left(v_{i}=d_{i}\right)$ iff $s\left(v_{i}\right)=d_{i}$ with $d_{i} \in D_{v_{i}}$.

- We have $s \models\left(v_{i}=s\left(v_{i}\right) \wedge \ldots \wedge v_{m}=s\left(v_{m}\right)\right)$.

- For $V^{\prime} \subseteq V$ and $s^{\prime} \in \operatorname{Eval}\left(V^{\prime}\right)$, we write

$$
s \models s^{\prime} \text { iff } \bigwedge_{v \in V^{\prime}} s\left(v_{i}\right)=s^{\prime}\left(v_{i}\right) .
$$

- For the logical formula $\alpha$ over the atomic propositions $\left(v_{i}=d_{i}\right)$, we write

$$
s \models \alpha \text { iff }\left(v_{i}=s\left(v_{i}\right) \wedge \ldots \wedge v_{m}=s\left(v_{m}\right)\right) \Longrightarrow \alpha .
$$


Given the pBN $\mathcal{B}=(V, W, X, \Theta)$, the topological order $\varrho$ on $V$, and the state $s \in \operatorname{States}(V)$, let $s\left[v_{i}=d_{i}\right]=s^{*}$, where

$-s^{*} \in \operatorname{States}(V)$,

$-s^{*}\left(v_{i}\right)=d_{i}$,

$-s^{*}(v)=s(v)$ for $v \neq v_{i}$ satisfying

$$
\varrho(v)<\varrho\left(v_{i}\right) \text { and } \exists c \in \operatorname{children}(v) \cdot \varrho(c)>\varrho\left(v_{i}\right) \text {, and }
$$

$-s^{*}(v)=*$ for all other variables.

Definition 3 (Evidence-agnostic pMC of pBN). Let $\mathcal{B}=(V, W, X, \Theta)$ be a $p B N$ and $\varrho=\left(v_{1}, \ldots, v_{m}\right)$ be a topological order over $V$. The $p M C$ of $\mathcal{B}$ is $\mathcal{M}_{\mathcal{B}}^{\varrho}=\left(S, s_{0}, X, P\right)$, where:

$-s_{0}=\left(v_{1}=*, \ldots, v_{m}=*\right)$ is the initial state,

$-S \subseteq$ States $(V)$ is the set of states defined as follows:

- $S=\bigcup_{0 \leq i \leq m} S_{i}$

- $S_{0}=\left\{\begin{array}{l}0 \leq i \leq m \\ \left.s_{0}\right\}\end{array}\right.$

- $S_{i+1}=\left\{s_{i}\left[v_{i+1}=d_{i+1}\right] \mid\right.$ for $s_{i} \in S_{i}$ and $\left.d_{i+1} \in D_{v_{i+1}}\right\}$ for $0 \leq i<m$

- and $P: S \times S \rightarrow[0,1]$ is the transition probability functions defined by the following two rules.

1. Let $0 \leq i<m, \overline{p a r} \in \operatorname{Eval}\left(\operatorname{parents}\left(v_{i+1}\right)\right)$, and $s_{i+1}=s_{i}\left[v_{i+1}=d_{i+1}\right] \in S_{i+1}$. For each $s_{i} \in S_{i}$ and $d_{i+1} \in D_{v_{i+1}}$,

$$
P\left(s_{i}, s_{i+1}\right)=\Theta(\overline{p a r})\left(d_{i+1}\right) \text { iff } s_{i} \models \overline{p a r} .
$$

2. $P\left(s_{m}, s_{m}\right)=1$ for each $s_{m} \in S_{m}$.

3. $P\left(s_{i}, s_{j}\right)=0$, otherwise.

The states generation in Definition 3 ensures that

$\forall 0 \leq i<m, \forall s_{i} \in S_{i} \cdot \exists ! \overline{p a r} \in \operatorname{Eval}\left(\operatorname{parents}\left(v_{i+1}\right)\right)$ such that $s_{i}=\overline{p a r}$.

Example. Fig. 2 (left) indicates the pMC obtained by Def. 3 for the pregnancy test $\mathrm{pBN}$ and the topological ordering $(P, U, B)$. The node names are abbreviated by their first letters and the "don't care" evaluations are omitted. The pMC starts in the initial state $s_{0}$, where all the variables are "don't care". The successor states of $s_{0}$ are determined by the first node in the topological ordering, i.e., Pregnancy $(P)$ : for each value in the domain of $P, s_{0}$ has a successor state. This yields the states $P=$ yes and $P=n o$ in the pMC. The transition probabilities from $s_{0}$ to these states naturally come from the the CPT of Pregnancy, see rule 1 . The states in the next leve 7 are subsequently determined by the node Urine Test. The transition probability, e.g., from the state $P=n o$ to the state $(P=n o, U=n e g)$ is determined by the corresponding CPT entry that is 0.893 . Note that the value of pregnancy is still kept at this level as $P$ still has an unprocessed child $(B)$. In the last level, the value of Pregnancy is forgotten as there is no children of $P$ remaining in the topological order.

\footnotetext{
${ }^{7}$ Note that Def. 3 imposes no backward edges: (a) $P\left(s_{i}, s_{j}\right)>0$ iff $s_{i} \in S_{i}$ and $s_{j} \in S_{i+1}$ or $i=j=m$, (b) $S_{i} \cap S_{j}=\emptyset$ for $i \neq j$. This allows us to consider levels for $\mathcal{M}_{\mathcal{B}}^{\varrho}$ : we refer to the states in $S_{i}$ as the states in the $i$ 'th level of the pMC.
} 

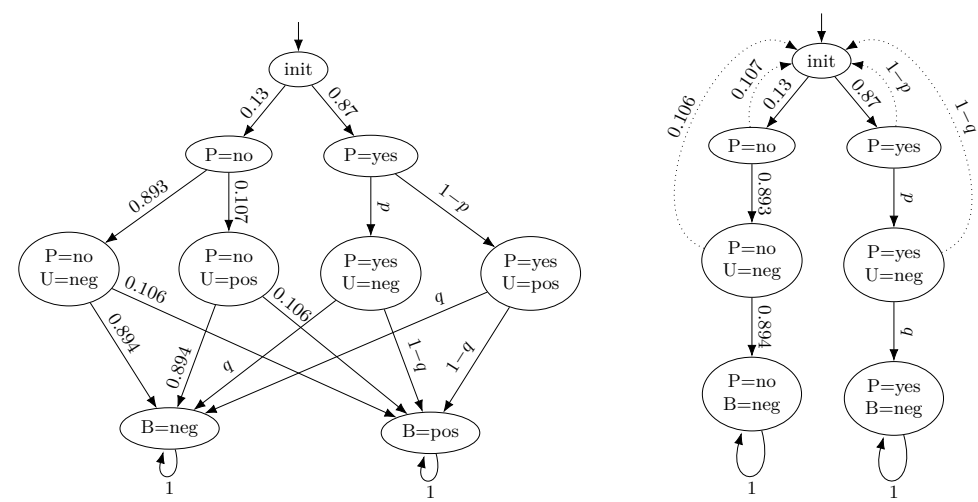

Fig. 2: The generated pMCs for the pregnancy test example based on (left) $\mathrm{pBN} 2 \mathrm{pMC}$ and (right) evidence-guided pBN2pMC transformation.

The following result relates (conditional) inference in pBNs to (conditional) reachability objectives in pMCs. The proof is provided in App. A

Proposition 1. Let $\mathcal{B}$ be a $p B N$ and $\varrho$ be a topological ordering on $V$. Let $E$ and $H$ be the evidence and the hypothesis. Then, for the $p M C \mathcal{M}_{\mathcal{B}}^{\varrho}$ obtained from Def. 3 we have:

$$
\operatorname{Pr}_{\mathcal{B}}(E)=1-\operatorname{Pr}_{\mathcal{M}_{\mathcal{B}}^{\rho}}(\diamond \neg E) \quad \text { and } \quad \operatorname{Pr}_{\mathcal{B}}(H \mid E)=\frac{1-\operatorname{Pr}_{\mathcal{M}_{\mathcal{B}}^{\rho}}(\diamond(\neg H \vee \neg E))}{1-\operatorname{Pr}_{\mathcal{M}_{\mathcal{B}}^{\rho}}(\diamond \neg E)},
$$

where the latter equality requires $\operatorname{Pr}_{\mathcal{B}}(\neg E)<1$.

This result directly enables to use techniques for feasibility checking on pMCs to $\mathrm{pBNs}$, and the use of techniques to compute reachability functions on pMCs to computing sensitivity functions on pBNs.

An evidence-tailored $p B N 2 p M C$ transformation. The above transformation is agnostic from the inference query. We now construct a pMC $\mathcal{M}_{\mathcal{B}, E}^{\varrho}$ tailored to a given evidence $E$. The transformation is inspired by a transformation on MCs [3] for computing conditional reachability probabilities. Let $\mathcal{B}=(V, E, X, \Theta)$ be a $\mathrm{pBN}$ and $\varrho$ be a topological order on $V$. Let $E=\left(v_{E_{1}}=d_{E_{1}}\right) \wedge \cdots \wedge\left(v_{E_{k}}=d_{E_{k}}\right)$ be the evidence defined as before. We construct pMC $\mathcal{M}_{\mathcal{B}, E}^{\varrho}$ by the following two amendments on the pMC $\mathcal{M}_{\mathcal{B}}^{\varrho}$ as defined in Def. 3

- Propagation operation: For $v_{j} \notin \operatorname{vars}(E)$ with $\varrho\left(v_{j}\right)<\varrho\left(v_{E_{k}}\right)$, we propagate the values of $v_{j}$ until the level $\varrho\left(v_{E_{k}}\right)$.

- Redirection operation: Let $\mathcal{M}_{\mathcal{B}, \varrho}^{\downarrow}=\left(S^{\downarrow}, s_{0}^{\downarrow}, X, P^{\downarrow}\right)$ be the pMC obtained from applying the propagation operation on $\mathcal{M}^{\varrho}$. Let $S_{\neg E}=\left\{s^{\downarrow} \in S^{\downarrow} \mid s^{\downarrow} \models\right.$ $\left(v_{E_{i}}=\neg d_{E_{i}}\right)$ for some $1 \leq i \leq k$ and $\left.\neg d_{E_{i}} \neq d_{E_{i}}\right\}$ be the set of sates in $\mathcal{M}_{\mathcal{B}, \varrho}^{\downarrow}$ that violate the evidence $E$. We reroute the direct transitions to the states in $S_{\neg E}$ to the initial state $s_{0}$ and delete the states in $S_{\neg E}$. 
Let $H$ be a logical formula over the atomic propositions $\left(v_{H_{i}}=d_{H_{i}}\right)$ that is decomposable to the sub-formulas $H_{\text {before }}$ and $H_{\text {after }}$, such that the variables appearing in $H_{\text {before }}$ all occur before $v_{E_{\text {last }}}$ in the topological order $\varrho$ and the variables involved in $H_{\text {after }}$ all occur after $v_{E_{\text {last }}}$. Without loss of generality, let $H=$ $\left(v_{H_{1}}=d_{H_{1}}\right) \wedge \cdots \wedge\left(v_{H_{l}}=d_{H_{l}}\right)$ be the hypothesis. Let $H_{\text {before }}=\bigwedge_{i=1}^{b}\left(v_{H_{i}}=d_{H_{i}}\right)$ with $v_{H_{i}}<\varrho v_{E_{\text {last }}}$ and let $H_{\text {after }}=\bigwedge_{i=a}^{l}\left(v_{H_{i}}=d_{H_{i}}\right)$ with $v_{H_{i}}>_{\varrho} v_{E_{\text {last }}}$, such that $H=H_{\text {before }} \wedge H_{\text {after }}$.

Proposition 2. For the evidence-tailored $p M C \mathcal{M}_{\mathcal{B}, E}^{\varrho}$ of $p B N \mathcal{B}$, we have:

$$
\operatorname{Pr}_{\mathcal{B}}(H \mid E)=1-\operatorname{Pr}_{\mathcal{M}_{\mathcal{B}, E}^{\varrho}}\left(\diamond\left(\left(\neg H_{\text {before }} \wedge E_{\text {last }}\right) \vee \neg H_{\text {after }}\right)\right) .
$$

This result facilitates using $\mathrm{pMC}$ techniques for $\mathrm{pBN}$ parameter tuning. For the formal definitions and the proof, see App. A.

Example. Fig. 2 (right) indicates the evidence-guided pMC generated for our running example, the ordering $(P, U, B)$, and the evidence $U=n e g \wedge B=n e g$. By the propagation operation the value of $P$ is propagated until the level of last evidence node $B$. By the redirection operation, the transitions leading to $U=p o s$ and $B=$ pos are redirected to the initial state. By Proposition 2

$$
\operatorname{Pr}_{\mathcal{B}}(P=\text { yes } \mid U=n e g \wedge B=n e g)=1-\operatorname{Pr}_{\mathcal{M}_{\mathcal{B}, E}}(\diamond(P=n o \wedge B=n e g)) .
$$

Ratio and difference parameter tuning by parameter lifting. The ratio problem on pBN $\mathcal{B}$ corresponds to finding an instantiation $u$ in the pMC $\mathcal{M}_{\mathcal{B}, E}^{\varrho}$ s.t.

$$
\operatorname{Pr}_{\mathcal{M}_{\mathcal{B}, E}^{\varrho}}[u](\diamond T) \geq q \cdot \operatorname{Pr}_{\mathcal{M}_{\mathcal{B}, E}^{\varrho}}[u](\diamond G),
$$

where $\operatorname{Pr}(\diamond T)$ stands for $1-\operatorname{Pr}_{\mathcal{M}_{\mathcal{B}, E}^{o}}[u]\left(\diamond\left(H=\neg h^{\prime} \vee E=\neg e\right)\right)$ and $\operatorname{Pr}(\diamond G)$ abbreviates $1-\operatorname{Pr}_{\mathcal{M}_{\mathcal{B}, E}^{e}}[u](\diamond(H=\neg h \vee E=\neg e))$. The problem can be solved using PLA: let region $R \subseteq \mathbb{R}_{\geq 0}^{n}$. We perform PLA for reaching $G$ and reaching $T$ on $\mathcal{M}_{\mathcal{B}}$, respectively. This gives upper $\left(U B_{T}, U B_{G}\right)$ and lower bounds ( $L B_{T}$, $\left.L B_{G}\right)$ for the probabilities on the left- and the right-hand side of $(2)$. Then:

- If $L B_{T} \geq q \cdot U B_{G}$, the region $R$ is accepting for the ratio property.

- If $L B_{T} \leq q \cdot U B_{G}$, the region $R$ is rejecting for the ratio property.

- Otherwise, refine the region $R$.

For difference parameter tuning, we adopt the above recipe by replacing 2 by:

$$
\operatorname{Pr}_{\mathcal{M}_{\mathcal{B}, E}^{\stackrel{\rho}{0}}}[u](\diamond T) \geq q+\operatorname{Pr}_{\mathcal{M}_{\mathcal{B}, E}^{\varrho}}[u](\diamond G) .
$$

\section{$5 \quad$ Experiments}

Our pBN analysis tool. We developed a prototypical tool on top of the tools Storm [25] and Prophesy [24], see Fig. 3 Storm is a probabilistic model checker 


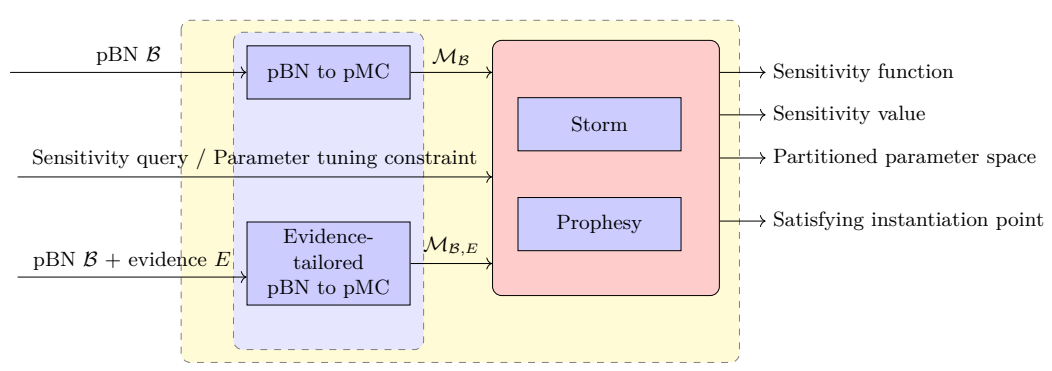

Fig. 3: Our prototypical tool-chain for synthesis problems on pBNs

that dominated the last (and only) two model-checking competitions, see qcomp. org Prophesy is an efficient tool for pMC synthesis. Our tool deploys pMC parameter synthesis techniques to analyze pBNs. It includes both $\mathrm{pBN} 2 \mathrm{pMC}$ transformations where $\mathrm{pBNs}$ are provided in an extended bif format. The pMCs are either encoded in Jani [8] or in the explicit drn format. It is also possible to transform non-parametric BNs into MCs and parameterize the MC. Storm is used to compute the sensitivity function and for parameter tuning using PLA. Prophesy is exploited for feasibility checking: find a parameter instance satisfying an inference query. Our tool-chain supports $p_{*} c_{*} r_{*}$, the general pBNs class. As baseline we used two synthesis tools for parametric BNs: SamIam and Bayesserver.

SamIam. SamIam ${ }^{8}$ is a commonly used tool for the sensitivity analysis for pBNs, developed at Darwiche's group at UCLA. It allows the specification of conditional, hypothesis ratio, and hypothesis difference constraints on pBNs. SamIam then attempts to identify minimal parameter changes that are necessary to satisfy these constraints. It supports the $\mathrm{pBN}$ classes $p_{1} c_{1} r_{1}$ and $p_{*} c_{1} r_{1}$.

Bayesserver. Bayeserver 9 is a commercial tool that offers sensitivity analysis and parameter tuning of pBNs. For sensitivity analysis, it computes the sensitivity function and sensitivity value. It also performs minimal-change parameter tuning for conditional, hypothesis ratio, and hypothesis difference constraints. It supports the classes $p_{1} c_{1} r_{1}$ and $p_{2} c_{\leq 2} r_{1}$ for sensitivity analysis and the class $p_{1} c_{1} r_{1}$ for parameter tuning. Tab. 1 lists the functionalities of all tools.

Experimental set-up. We took benchmarks from [45] and conducted all our experiments on a $2.3 \mathrm{GHz}$ Intel Core i5 processor with $16 \mathrm{~GB}$ of RAM. We focused on questions such as:

1. What is the scalability for computing sensitivity functions on pBNs?

2. What is the practical scalability for feasibility checking?

3. To what extent is PLA applicable to parameter tuning for pBNs?

Computing sensitivity function. We performed a series of experiments for computing pBN sensitivity functions using our tool-chain for the $p_{*} c_{*} r_{1}$ class. Fig. 4

8 http://reasoning.cs.ucla.edu/samiam

9 https://www.bayesserver.com 
Table 1: Overview of the capabilities of the pBN synthesis tools considered.

\begin{tabular}{|l||c|c|c|}
\hline & SamIam & Bayesserver & Storm-Prophesy \\
\hline \hline computing sensitivity function & $\boldsymbol{X}$ & $p_{\leq 2} c_{\leq 2} r_{1}$ & $p_{*} c_{*} r_{*}$ \\
\hline computing sensitivity value & $\boldsymbol{X}$ & $p_{\leq 2} c_{\leq 2} r_{1}$ & $p_{1} c_{*} r_{*}$ \\
\hline simple parameter tuning & $p_{*} c_{1} r_{1}$ & $p_{1} c_{1} r_{1}$ & $p_{*} c_{*} r_{*}$ \\
\hline difference parameter tuning & $p_{*} c_{1} r_{1}$ & $p_{1} c_{1} r_{1}$ & $p_{*} c_{*} r_{*}$ \\
\hline ratio parameter tuning & $p_{*} c_{1} r_{1}$ & $p_{1} c_{1} r_{1}$ & $p_{*} c_{*} r_{*}$ \\
\hline minimal change tuning & $p_{*} c_{1} r_{1}$ & $p_{1} c_{1} r_{1}$ & $p_{*} c_{*} r_{*}$ \\
\hline
\end{tabular}

summarizes the results. The $x$-axis (log scale) indicates the pBN benchmarks and the $y$-axis denotes the timing in seconds. The numbers on the bars indicate the number of parameters in the solution functions, which is related to the number of relevant parameters identified for the given query. We observe that Storm scales up to 380 parameters for very large networks such as hailfinder. The blue bars represent regular computations, while the orange bars indicate the impact of bisimulation minimization, a built-in reduction technique in Storm.

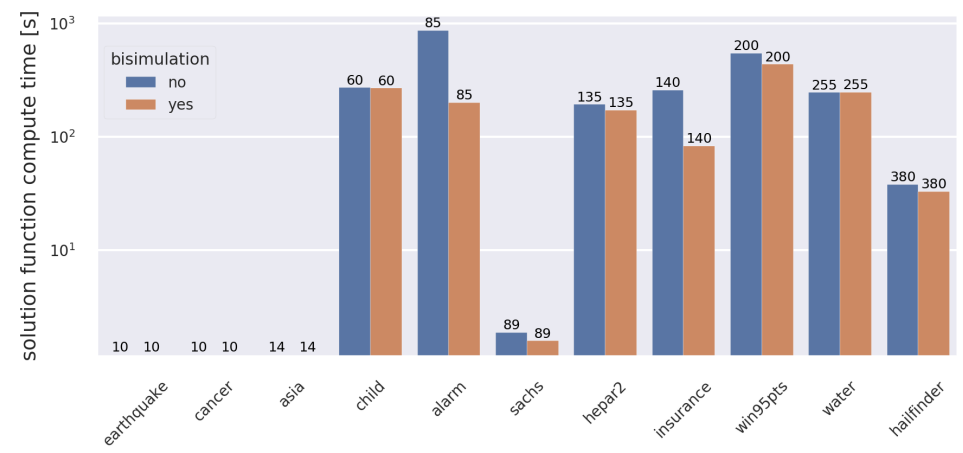

Fig. 4: Storm's performance for calculating prior sensitivity functions of pBNs. ${ }^{10}$

Feasibility checking. Our tool exploits Prophesy to find a parameter instantiation $u$ of $\mathrm{pBN} \mathcal{B}$ such that the $\mathrm{BN} \mathcal{B}[u]$ satisfies the given inference query. We have performed a set of experiments for the class $p_{*} c_{*} r_{1}$. Fig. 5 ( $\log -\log$ scale) illustrates the results; the $x$-axis indicates the number of parameters in the $\mathrm{pBN}$ and the $y$-axis the time for feasibility checking (in seconds). Each line corresponds to a $\mathrm{pBN}$ and the points on the lines represent single experiments. We inserted the parameters in the predecessors of the query nodes (i.e., in $H$ ) to maximize

${ }^{10}$ A comparison with the other tools was not applicable, as SamIam does not explicitly offers sensitivity function computation and Bayesserver sensitivity analysis is limited to 1 or 2 parameters, see Tab. 1 . 

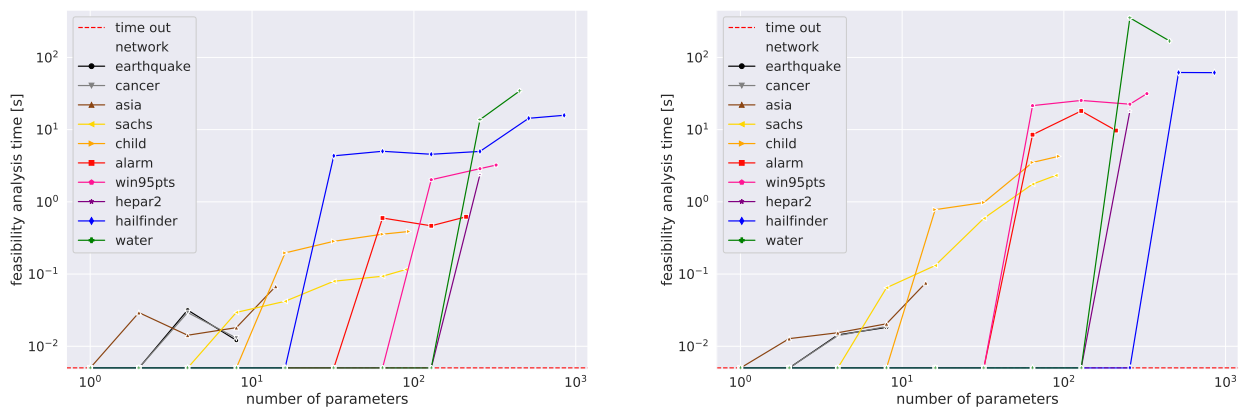

Fig. 5: Feasibility checking on pBN benchmarks by (left) QCQP and (right) PSO.

their relevance. We also imposed queries over multiple nodes at once to push the boundaries. We used convex optimization (QCQP ${ }^{11}$ ) (left plot) and PSO (right plot). Prophesy was able to handle up to 853 parameters.

Approximate parameter synthesis on pBNs: Tuning the parameters and more. Experiments on the pBN benchmarks using PLA aimed at (a) the classes $p_{1} c_{1} r_{1}$ and $p_{*} c_{1} r_{1}$ to validate them against SamIam and Bayesserver, and (b) the class $p_{*} c_{*} r_{*}$ to investigate the potential of PLA for general pBNs. Fig. 6 visualizes the results for the alarm $\mathrm{pBN}$ with 2 parameters occurring in 26 rows of $3 \mathrm{CPTs}$, i.e., a pBN with parameter dependencies. The parameter $x$ was used in the CPT entries $e_{1}, \cdots, e_{k}$ only when the probability values of those entries coincided in the original $\mathrm{BN}$. As seen in the figure, PLA can partition the entire $n$-way parameter space. The minimal-change parameter values can be extracted from the PLA results, where the precision depends on the PLA approximation factor.
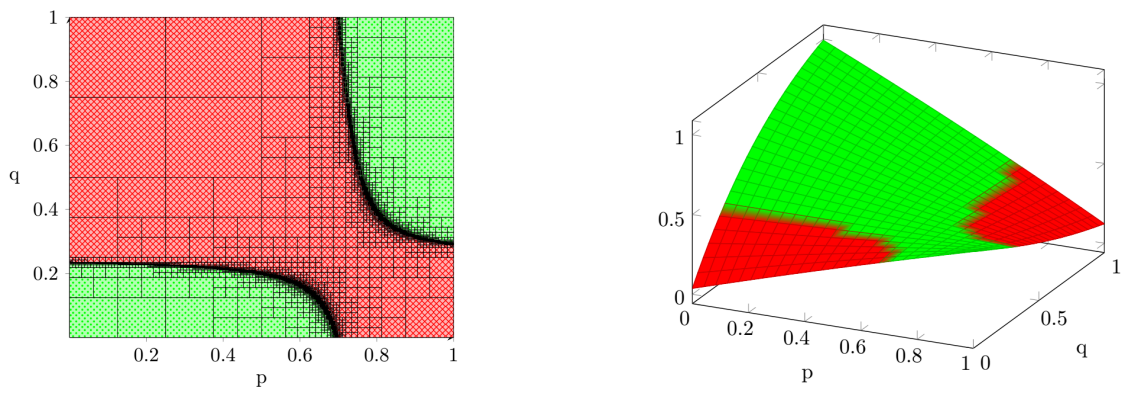

Fig. 6: PLA results on the alarm $\mathrm{pBN}\left(p_{2} c_{3} r_{26}\right)$ for the constraint $\operatorname{Pr}($ venttube $=$ $0 \mid$ ventlung $=0)>0.6$ with a $99 \%$ parameter space coverage.

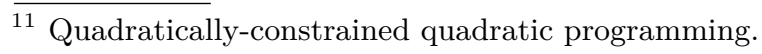




\section{Conclusion}

This paper exploited tools and techniques for parameter synthesis on Markov chains to synthesis problems on parametric Bayesian networks. Prototypical tool support for $\mathrm{pBN}$ analysis on top of existing pMC synthesis tools has been realized. Our experiments indicate that pMC techniques can scale sensitivity analysis and parameter tuning tasks on pBNs. The experiments reveal the potential of parameter lifting 41 for partitioning the parameter space of pBNs. Most importantly, the proposed techniques are applicable to general pBNs - no restrictions are imposed on the number or occurrence of parameters - and may involve parameter dependencies. Future work include finding optimal parameter settings [47, exploiting monotonicity checking [46] and to extend the current work to (parametric) dynamic, Gaussian [12], and recursive BNs [32].

Acknowledgement. We thank Robin Drahovsky for his contributions on transforming pMCs into pBNs, Caroline Jabs for her implementation efforts, and Sebastian Junges, Tim Quatmann, and Matthias Volk for discussions. We also thank Arthur Choi for his support.

\section{References}

1. Baier, C., Hensel, C., Hutschenreiter, L., Junges, S., Katoen, J., Klein, J.: Parametric Markov chains: PCTL complexity and fraction-free Gaussian elimination. Inf. Comput. 272, 104504 (2020)

2. Baier, C., Katoen, J.: Principles of Model Checking. MIT Press (2008)

3. Baier, C., Klein, J., Klüppelholz, S., Märcker, S.: Computing conditional probabilities in Markovian models efficiently. In: TACAS. Lecture Notes in Computer Science, vol. 8413, pp. 515-530. Springer (2014)

4. Bartocci, E., Grosu, R., Katsaros, P., Ramakrishnan, C.R., Smolka, S.A.: Model repair for probabilistic systems. In: TACAS. LNCS, vol. 6605. Springer (2011)

5. Bartocci, E., Kovács, L., Stankovic, M.: Analysis of Bayesian networks via ProbSolvable Loops. In: ICTAC. Lecture Notes in Computer Science, vol. 12545, pp. 221-241. Springer (2020)

6. Beaumont, P., Huth, M.: Constrained Bayesian networks: Theory, optimization, and applications. CoRR abs/1705.05326 (2017)

7. Bolt, J.H., van der Gaag, L.C.: Balanced tuning of multi-dimensional Bayesian network classifiers. In: ECSQARU. Lecture Notes in Computer Science, vol. 9161, pp. 210-220. Springer (2015)

8. Budde, C.E., Dehnert, C., Hahn, E.M., Hartmanns, A., Junges, S., Turrini, A.: JANI: quantitative model and tool interaction. In: TACAS (2). Lecture Notes in Computer Science, vol. 10206, pp. 151-168 (2017)

9. Castillo, E.F., Gutiérrez, J.M., Hadi, A.S.: Parametric structure of probabilities in Bayesian networks. In: ECSQARU. Lecture Notes in Computer Science, vol. 946, pp. 89-98. Springer (1995)

10. Castillo, E.F., Gutiérrez, J.M., Hadi, A.S.: Goal oriented symbolic propagation in Bayesian networks. In: AAAI/IAAI, Vol. 2. pp. 1263-1268. AAAI Press / The MIT Press (1996) 
11. Castillo, E.F., Gutiérrez, J.M., Hadi, A.S.: Sensitivity analysis in discrete Bayesian networks. IEEE Trans. Syst. Man Cybern. Part A 27(4), 412-423 (1997)

12. Castillo, E.F., Gutiérrez, J.M., Hadi, A.S., Solares, C.: Symbolic propagation and sensitivity analysis in Gaussian Bayesian networks with application to damage assessment. Artif. Intell. Eng. 11(2), 173-181 (1997)

13. Chan, H., Darwiche, A.: When do numbers really matter? J. Artif. Intell. Res. 17, 265-287 (2002)

14. Chan, H., Darwiche, A.: Sensitivity analysis in Bayesian networks: From single to multiple parameters. In: UAI. pp. 67-75. AUAI Press (2004)

15. Chan, H., Darwiche, A.: A distance measure for bounding probabilistic belief change. Int. J. Approx. Reason. 38(2), 149-174 (2005)

16. Chen, T., Hahn, E.M., Han, T., Kwiatkowska, M.Z., Qu, H., Zhang, L.: Model repair for Markov decision processes. In: TASE. IEEE (2013)

17. Coupé, V.M.H., van der Gaag, L.C.: Properties of sensitivity analysis of Bayesian belief networks. Ann. Math. Artif. Intell. 36(4), 323-356 (2002)

18. Coupe, V.M., van der Gaag, L.C.: Practicable sensitivity analysis of Bayesian belief networks, vol. 1998. Utrecht University: Information and Computing Sciences (1998)

19. Coupé, V.M., van der Gaag, L.C., Habbema, J.D.F.: Sensitivity analysis: an aid for belief-network quantification. The Knowledge Engineering Review 15(3), 215-232 (2000)

20. Cubuktepe, M., Jansen, N., Junges, S., Katoen, J.P., Papusha, I., Poonawala, H.A., Topcu, U.: Sequential convex programming for the efficient verification of parametric MDPs. In: TACAS. LNCS, vol. 10206, pp. 133-150 (2017)

21. Cubuktepe, M., Jansen, N., Junges, S., Katoen, J., Topcu, U.: Synthesis in pMDPs: A tale of 1001 parameters. In: ATVA. Lecture Notes in Computer Science, vol. 11138, pp. 160-176. Springer (2018)

22. Darwiche, A.: Modeling and Reasoning with Bayesian Networks. Cambridge University Press (2009)

23. Daws, C.: Symbolic and parametric model checking of discrete-time Markov chains. In: ICTAC. Lecture Notes in Computer Science, vol. 3407, pp. 280-294. Springer (2004)

24. Dehnert, C., Junges, S., Jansen, N., Corzilius, F., Volk, M., Bruintjes, H., Katoen, J., Ábrahám, E.: Prophesy: A probabilistic parameter synthesis tool. In: CAV (1). Lecture Notes in Computer Science, vol. 9206, pp. 214-231. Springer (2015)

25. Dehnert, C., Junges, S., Katoen, J., Volk, M.: A storm is coming: A modern probabilistic model checker. In: CAV (2). Lecture Notes in Computer Science, vol. 10427, pp. 592-600. Springer (2017)

26. Druzdzel, M.J., van der Gaag, L.C.: Building probabilistic networks:" where do the numbers come from?". IEEE Trans. Knowl. Data Eng. 12(4), 481-486 (2000)

27. Fang, X., Calinescu, R., Gerasimou, S., Alhwikem, F.: Fast parametric model checking through model fragmentation. In: ICSE. pp. 835-846. IEEE (2021)

28. Fang, X., Calinescu, R., Gerasimou, S., Alhwikem, F.: Fast parametric model checking through model fragmentation. CoRR abs/2102.01490 (2021)

29. Van der Gaag, L.C., Renooij, S., Coupé, V.M.: Sensitivity analysis of probabilistic networks. In: Advances in probabilistic graphical models, pp. 103-124. Springer (2007)

30. Gainer, P., Hahn, E.M., Schewe, S.: Accelerated model checking of parametric Markov chains. In: ATVA. Lecture Notes in Computer Science, vol. 11138, pp. 300-316. Springer (2018) 
31. Hahn, E.M., Hermanns, H., Zhang, L.: Probabilistic reachability for parametric Markov models. Int. J. Softw. Tools Technol. Transf. 13(1), 3-19 (2011)

32. Jaeger, M.: Complex probabilistic modeling with recursive relational Bayesian networks. Ann. Math. Artif. Intell. 32(1-4), 179-220 (2001)

33. Jansen, N., Corzilius, F., Volk, M., Wimmer, R., Ábrahám, E., Katoen, J., Becker, B.: Accelerating parametric probabilistic verification. In: QEST. Lecture Notes in Computer Science, vol. 8657, pp. 404-420. Springer (2014)

34. Jensen, F.V.: Gradient descent training of Bayesian networks. In: ESCQARU. Lecture Notes in Computer Science, vol. 1638, pp. 190-200. Springer (1999)

35. Junges, S., Ábrahám, E., Hensel, C., Jansen, N., Katoen, J., Quatmann, T., Volk, M.: Parameter synthesis for Markov models. CoRR abs/1903.07993 (2019)

36. Junges, S., Katoen, J.P., Pérez, G.A., Winkler, T.: The complexity of reachability in parametric Markov decision processes. J. Comput. Syst. Sci. 119, 183-210 (2021)

37. Kjærulff, U., van der Gaag, L.C.: Making sensitivity analysis computationally efficient. In: UAI. pp. 317-325. Morgan Kaufmann (2000)

38. Kwisthout, J., van der Gaag, L.C.: The computational complexity of sensitivity analysis and parameter tuning. In: UAI. pp. 349-356. AUAI Press (2008)

39. Lanotte, R., Maggiolo-Schettini, A., Troina, A.: Parametric probabilistic transition systems for system design and analysis. Formal Aspects Comput. 19(1), 93-109 (2007)

40. Laskey, K.B.: Sensitivity analysis for probability assessments in Bayesian networks. IEEE Trans. Syst. Man Cybern. 25(6), 901-909 (1995)

41. Quatmann, T., Dehnert, C., Jansen, N., Junges, S., Katoen, J.P.: Parameter synthesis for Markov models: Faster than ever. In: ATVA. LNCS, vol. 9938 (2016)

42. Renooij, S.: Co-variation for sensitivity analysis in Bayesian networks: Properties, consequences and alternatives. Int. J. Approx. Reason. 55(4), 1022-1042 (2014)

43. Salmani, B., Katoen, J.: Bayesian inference by symbolic model checking. In: QEST. Lecture Notes in Computer Science, vol. 12289, pp. 115-133. Springer (2020)

44. Salmani, B., Katoen, J.: Fine-tuning the odds in bayesian networks. CoRR abs/2105.14371 (2021)

45. Scutari, M.: Bayesian network repository. https://www.bnlearn.com, accessed: 2019

46. Spel, J., Junges, S., Katoen, J.: Are parametric Markov chains monotonic? In: ATVA. Lecture Notes in Computer Science, vol. 11781, pp. 479-496. Springer (2019)

47. Spel, J., Junges, S., Katoen, J.: Finding provably optimal Markov chains. In: TACAS (1). Lecture Notes in Computer Science, vol. 12651, pp. 173-190. Springer (2021)

48. Tolo, S., Patelli, E., Beer, M.: An open toolbox for the reduction, inference computation and sensitivity analysis of Credal networks. Adv. Eng. Softw. 115, 126-148 (2018) 


\section{A Inference query correspondence}

Here, we provide the proofs for Proposition 1 and Proposition 2 The results are formalized for non-parametric MCs of BNs and carry over to pMCs and pBNs in a straightforward manner.

Preliminaries and notations. Let $\mathcal{M}=\left(S, s_{0}, P\right)$ be a $\mathrm{MC}$, Paths $(\mathcal{M})$ be the set of paths in $\mathcal{M}$ that start in the initial state $s_{0}$, and $\operatorname{Paths}^{*}(\mathcal{M})$ the set of their finite prefixes. For the path $\pi=s_{0} \ldots s_{n} \in \operatorname{Path}^{*}(\mathcal{M})$, let $P(\pi)=\prod_{i=0}^{n-1} P\left(s_{i}, s_{i+1}\right)$. For infinite paths, a probability measure Pr is defined using a standard cylinder set construction [2, Ch. 10]. Let pre $(s)$ be the direct predecessors of $s$ and $\operatorname{pre}^{*}(s)$ be its reflexive and transitive closure. Let $G \subseteq S$ be the set of goal states in $\mathcal{M}$ and $\diamond G$ denote the set of paths in $\mathcal{M}$ that eventually reach $G$, i.e., $\diamond G=\{\pi \in$ $\operatorname{Paths}(\mathcal{M}) \mid \exists i \in \mathbb{N} . \pi(i) \in G\}$. Let $\operatorname{Paths}(\mathcal{M}, \diamond G)=\operatorname{Paths}(\mathcal{M}) \cap(S \backslash G)^{*} G$, and

$$
\underset{\mathcal{M}}{\operatorname{Pr}}(\diamond G)=\sum_{s_{0} \ldots s_{n} \in \operatorname{Paths}(\mathcal{M}, \diamond G)} P\left(s_{0} \ldots s_{n}\right) .
$$

For each $\mathrm{MC}, \operatorname{Pr}(\diamond G)$ is measurable. Let variable $p_{s}=\operatorname{Pr}_{\mathcal{M}}(s \models \diamond G)$ denote the probability to reach $G$ from state $s$. It follows that

- if $G$ is not reachable from $s$, then $p_{s}=0$,

- if $s \in G$, then $p_{s}=1$, and

- For any state $s \in \operatorname{pre}^{*}(G) \backslash G$,

$$
p_{s}=\underbrace{\sum_{t \in S \backslash G} P(s, t) \cdot p_{t}}_{\text {reaching } G \text { via state } t}+\underbrace{\sum_{u \in G} P(s, u)}_{\text {reaching } G \text { in one step }} .
$$

This yields a system of linear equations, by which $\operatorname{Pr}_{\mathcal{M}}(\diamond G)=p_{s_{0}}$.

Let $\square G$ denote the set of paths in $\mathcal{M}$ that always satisfy $G$, i.e.,

$$
\begin{gathered}
\square G \equiv \neg \diamond \neg G \text {. Thus } \\
\operatorname{Pr}(\square G)=1-\operatorname{Pr}(\diamond \neg G) .
\end{gathered}
$$

Let $\mathrm{BN} \mathcal{B}=(V, W, \Theta)$ and the topological order $\varrho=\left(v_{1}, \cdots, v_{m}\right)$ on $V$ be given. Let $E$ be the evidence in the form of $E=E_{1} \wedge \ldots \wedge E_{k}=\left(v_{E_{1}}=\right.$ $\left.d_{E_{1}}\right) \wedge \ldots \wedge\left(v_{E_{k}}=d_{E_{k}}\right)$ with $v_{E_{i}}<_{\varrho} v_{E_{i+1}}$. Recall that we often denote $E_{k}$ as $E_{\text {last }}$. Let $\mathcal{M}_{\mathcal{B}}^{\varrho}=\left(S, s_{0}, P\right)$ be the evidence-agnostic $\mathrm{MC}$ of $\mathrm{BN} \mathcal{B}$ as defined in Def. 3 Let $\mathcal{M}_{\mathcal{B}, E}^{\varrho}$ denote the evidence-tailored $\mathrm{MC}$ of the $\mathrm{BN} \mathcal{B}$. 
Query correspondence for the evidence-agnostic MC. We prove Proposition 1 by indicating the strong correspondence between the paths of the evidence-agnostic MC of $\mathcal{B}$ and the tree-like MC [43] of $\mathcal{B}$. Let $\mathcal{M}_{\mathcal{B}}^{\varrho}=\left(\boldsymbol{S}, \boldsymbol{s}_{\mathbf{0}}, \boldsymbol{P}\right)$ be the tree-like $\mathrm{MC}$ of $\mathcal{B}$ given the topological order $\varrho$. The construction of $\mathcal{M}_{\mathcal{B}}^{\varrho} 43$ is similar to that of $\mathcal{M}_{\mathcal{B}}^{\varrho}$ and only deviates in the following. Let $s_{i-1}$ be a state at the $i-1$ 'th level of $\mathcal{M}_{\mathcal{B}}^{\mathcal{O}}$. The successor states of $\boldsymbol{s}_{\boldsymbol{i}-\mathbf{1}}$ are obtained for each $d_{i} \in D_{v_{i}}$ by $\boldsymbol{s}_{\boldsymbol{i}-1}\left[v_{i}=d_{i}\right]=\boldsymbol{s}$, where

$-\boldsymbol{s}\left(v_{i}\right)=d_{i}$ and

$-\boldsymbol{s}(v)=\boldsymbol{s}_{\boldsymbol{i - 1}}(v)$ for $v \neq v_{i}$.

The transition probabilities are determined analogously to that of $\mathcal{M}_{\mathcal{B}}^{\varrho}$. This treelike $\mathrm{MC}$ is thus obtained by considering all possible valuations of the variables in $\mathrm{BN} \mathcal{B}$ adhering to the topological order $\varrho$. Inference on the $\mathrm{BN} \mathcal{B}$ can be reduced to computing reachability probabilities on the tree-like $\mathrm{MC} \mathcal{M}_{\mathcal{B}}^{\varrho}$, as

$$
\operatorname{Pr}_{\mathcal{B}}(E)=\operatorname{Pr}_{\mathcal{M}_{\mathcal{B}}^{o}}(\diamond E) \quad \text { and } \quad \operatorname{Pr}_{\mathcal{B}}(H \wedge E)=\operatorname{Pr}_{\mathcal{M}_{\mathcal{B}}^{\rho}}(\diamond(H \wedge E)) .
$$

This is formally shown in [43].

Let $A \subseteq V$ be a subset of $\mathrm{BN}$ variables and $\eta \in \operatorname{Eval}(A)$ be an evaluation of variables in $A$. Let Paths $\left(\mathcal{M}_{\mathcal{B}}^{\varrho}, \diamond \eta\right)=s_{0} s_{1} \ldots \in \operatorname{Paths}\left(\mathcal{M}_{\mathcal{B}}^{\varrho}\right)$ such that $\exists \boldsymbol{s}_{\boldsymbol{k}}$ with $\boldsymbol{s}_{\boldsymbol{k}}(v)=\eta(v)$ for all $v \in A$. Let $\operatorname{Paths}\left(\mathcal{M}_{\mathcal{B}}^{\varrho}, \square \eta^{*}\right)=s_{0} s_{1} \ldots \in$ Paths $\left(\mathcal{M}_{\mathcal{B}}^{\varrho}\right)$ such that for each $s_{i}$ and each $v \in A, s_{i}(v)=\eta(v)$ or $s_{i}(v)=*$.

Lemma 1. Path $\pi=s_{0} s_{1} \ldots \in \operatorname{Paths}\left(\mathcal{M}_{\mathcal{B}}^{\varrho}, \square \eta^{*}\right)$ if and only if there is the corresponding path $\boldsymbol{\pi}=\boldsymbol{s}_{\mathbf{0}} \boldsymbol{s}_{\mathbf{1}} \ldots \in \operatorname{Paths}\left(\mathcal{M}_{\mathcal{B}}^{\varrho}, \diamond \eta\right)$ such that for each $i \in \mathbb{N}$,

$$
P\left(s_{i}, s_{i+1}\right)=\boldsymbol{P}\left(s_{\boldsymbol{i}}, \boldsymbol{s}_{\boldsymbol{i}+\mathbf{1}}\right) .
$$

Proof. We indicate the correspondence between the paths in one direction. The proof is analogous for the other direction. Let $\pi=s_{0} s_{1} \ldots \in \operatorname{Paths}\left(\mathcal{M}_{\mathcal{B}}^{\varrho}, \diamond \eta\right)$. By the definition of $\mathcal{M}_{\mathcal{B}}^{\varrho}$,

- $s_{0}$ is the initial state,

- for $1 \leq i \leq m, \boldsymbol{s}_{\boldsymbol{i}} \in \boldsymbol{S}_{\boldsymbol{i}}$ is state at the $i$ th level of $\boldsymbol{\mathcal { M }}_{\boldsymbol{B}}^{\mathrm{o}}$, and

- for each $\boldsymbol{s}_{\boldsymbol{i}} \in \boldsymbol{S}_{\boldsymbol{i}}, \exists ! d_{i} \in D_{v_{i}}$ such that $\boldsymbol{s}_{\boldsymbol{i}} \models\left(v_{i}=d_{i}\right){ }^{12}$

Let vals $_{\boldsymbol{\pi}}=d_{1} d_{2} \cdots d_{m}$ be the sequence of the values for BN variables along the path $\boldsymbol{\pi}$, where $\operatorname{vals}_{\boldsymbol{\pi}}(i)=d_{i} \in D_{v_{i}}$ iff $\boldsymbol{s}_{\boldsymbol{i}} \models\left(v_{i}=d_{i}\right)$. The corresponding path $\pi \in \operatorname{Paths}\left(\mathcal{M}_{\mathcal{B}}^{\varrho}, \square \eta^{*}\right)$ is then obtained as follows.

Let $0 \leq i<m$ and $s_{i} \in S_{i}$ be a state at the $i$ th level of $\mathcal{M}_{\mathcal{B}}^{\varrho}$. Def. 3 ensures that

- for each $d_{i+1} \in D_{v_{i+1}}, \exists ! s_{i+1} \in \operatorname{succ}\left(s_{i}\right)$ such that $s_{i+1} \models\left(v_{i+1}=d_{i+1}\right)$.

Given $\operatorname{val}_{\boldsymbol{\pi}}$, the path $\pi=s_{0} s_{1} \cdots \in \operatorname{Paths}\left(\mathcal{M}_{\mathcal{B}}^{\varrho}\right)$ is then obtained, where

- $s_{0}$ is the initial state of $\mathcal{M}_{\mathcal{B}}^{\varrho}$,

\footnotetext{
$\overline{12}$ Note that $v_{i}$ is the $i$ th variable in the topological order $\varrho$.
} 
- for $0 \leq i<m, s_{i+1}$ is the unique state in $\operatorname{succ}\left(s_{i}\right)$ with

$$
s_{i+1} \models\left(v_{i+1}=\operatorname{vals}_{\boldsymbol{\pi}}(i+1)\right),
$$

- and for $i \geq m, s_{i+1}=s_{m}$.

Note that for $0 \leq i<m, P\left(s_{i}, s_{i+1}\right)=\boldsymbol{P}\left(\boldsymbol{s}_{\boldsymbol{i}}, \boldsymbol{s}_{\boldsymbol{i}+\mathbf{1}}\right)=\Theta(\overline{p a r})\left(\operatorname{vals}_{\boldsymbol{\pi}}(i+1)\right)$ for the parent valuation $\overline{p a r} \in \operatorname{Eval}\left(\operatorname{parents}\left(v_{i+1}\right)\right)$ with $s_{i} \models \overline{p a r}$. For $i \geq m$, $P\left(s_{i}, s_{i+1}\right)=\boldsymbol{P}\left(s_{\boldsymbol{i}}, \boldsymbol{s}_{\boldsymbol{i}+\mathbf{1}}\right)=1$.

It remains to show that $\pi \in \operatorname{Paths}\left(\mathcal{M}_{\mathcal{B}}^{\varrho}, \square \eta^{*}\right)$ :

(a) Path $\boldsymbol{\pi}=\boldsymbol{s}_{0} \boldsymbol{s}_{\mathbf{1}} \cdots \in \operatorname{Paths}\left(\mathcal{M}_{\mathcal{B}}^{\varrho}, \diamond \eta\right)$. Thus, $\exists k$ such that $\boldsymbol{s}_{\boldsymbol{k}}(v)=\eta(v)$ for all the variables $v \in A$.

(b) The $\mathrm{BN}$ variable $v$ is not assigned to two distinct values in $D_{v}$ along a given path in $\operatorname{Paths}\left(\mathcal{M}_{\mathcal{B}}^{\boldsymbol{\varrho}}\right)$, i.e., for $d \in D_{v}$,

$$
\boldsymbol{s}_{\boldsymbol{k}}(v)=d \Longrightarrow \boldsymbol{s}_{\boldsymbol{i}}(v)=d \vee \boldsymbol{s}_{\boldsymbol{i}}(v)=* \text { for } i<k \text { and } i>k .
$$

(c) Let $\boldsymbol{s}_{\boldsymbol{i}} \in \boldsymbol{S}_{\boldsymbol{i}}$ be a state at the $i$ 'th level of $\mathcal{M}_{\mathcal{B}}^{\varrho}$ and $v_{i}$ be the $i$ 'th variable in the topological order. Then by the definition of $\mathcal{M}_{\mathcal{B}}^{\varrho}, \boldsymbol{s}_{\boldsymbol{i}}\left(v_{i}\right) \neq *$.

It follows by (a), (b), and (c) that $s_{i}\left(v_{i}\right)=$ val $_{\boldsymbol{\pi}_{\boldsymbol{\pi}}}(i)=\eta\left(v_{i}\right)$ for $v_{i} \in A$. Moreover, similarly to argument (b), it holds that for $d_{i} \in D_{v_{i}}$,

$$
s_{i}\left(v_{i}\right)=d_{i} \Longrightarrow s_{j}\left(v_{i}\right)=d_{i} \vee s_{j}\left(v_{i}\right)=* \text { for } j<i \text { and } j>i .
$$

It thus yields $\pi \in \operatorname{Paths}\left(\mathcal{M}_{\mathcal{B}}^{\varrho}, \square \eta^{*}\right)$. This ends the proof for Lemma 1

For the evidence $E=\bigwedge_{i=1}^{k}\left(v_{i}=d_{i}\right)$, let $E^{*}=\bigwedge_{i=1}^{k}\left(v_{i}=d_{i} \vee v_{i}=*\right)$, 13 The following equations are obtained by considering $\eta:=E$ and $\eta:=E \wedge H$ in Lemma 1

$$
\operatorname{Pr}_{\mathcal{M}_{\mathcal{B}}^{o}}(\diamond E)=\operatorname{Pr}_{\mathcal{M}_{\mathcal{B}}^{\rho}}\left(\square E^{*}\right) \quad \text { and } \quad \operatorname{Pr}_{\mathcal{M}_{\mathcal{B}}^{o}}(\diamond(H \wedge E))=\operatorname{Pr}_{\mathcal{M}_{\mathcal{B}}^{\rho}}\left(\square\left(H^{*} \wedge E^{*}\right)\right) .
$$

Proposition 1. For the pMC $\mathcal{M}_{\mathcal{B}}^{\varrho}$,

$$
\operatorname{Pr}_{\mathcal{B}}(E)=1-\operatorname{Pr}_{\mathcal{M}_{\mathcal{B}}^{\varrho}}(\diamond \neg E) \quad \text { and } \quad \operatorname{Pr}_{\mathcal{B}}(H \mid E)=\frac{1-\operatorname{Pr}_{\mathcal{M}_{\mathcal{B}}^{\varrho}}(\diamond(\neg H \vee \neg E))}{1-\operatorname{Pr}_{\mathcal{M}_{\mathcal{B}}^{\varrho}}(\diamond \neg E)},
$$

where the latter equality requires $\operatorname{Pr}_{\mathcal{B}}(\neg E)<1$.

Proof. It follows from equations (8) and (9) that

$$
\operatorname{Pr}_{\mathcal{B}}(E)=\operatorname{Pr}_{\mathcal{M}_{\mathcal{B}}^{\varrho}}\left(\square E^{*}\right) \quad \text { and } \operatorname{Pr}_{\mathcal{B}}(H \wedge E)=\operatorname{Pr}_{\mathcal{M}_{\mathcal{B}}^{\rho}}\left(\square\left(H^{*} \wedge E^{*}\right)\right) .
$$

Without loss of generality, assume that the BN variables in $V$ are binary-valued with $D_{v_{i}}=\left\{d_{i}, \neg d_{i}\right\}$. Definition 3 ensures that for all $s \in S, s \models\left(v_{i}=d_{i}\right) \vee\left(v_{i}=\right.$ $\left.\neg d_{i}\right) \vee\left(v_{i}=*\right)$. Let $\neg E=\bigvee_{i=1}^{k}\left(v_{i}=\neg d_{i}\right)$. It follows that

$$
\neg\left(E^{*}\right) \equiv \neg E, \quad \text { thus }
$$

$\overline{13} H^{*}$ is defined analogously. 


$$
\operatorname{Pr}_{\mathcal{M}_{\mathcal{B}}^{\varrho}}\left(\square E^{*}\right)=1-\operatorname{Pr}_{\mathcal{M}_{\mathcal{B}}^{o}}\left(\diamond \neg\left(E^{*}\right)\right)=1-\operatorname{Pr}_{\mathcal{M}_{\mathcal{B}}^{\varrho}}(\diamond \neg E) .
$$

Since $E$ allows the conjunction of multiple variables, equation 11 is analogously valid for $(E \wedge H)$, i.e.,

$$
\operatorname{Pr}_{\mathcal{M}_{\mathcal{B}}^{\varrho}}\left(\square\left(H^{*} \wedge E^{*}\right)\right)=1-\operatorname{Pr}_{\mathcal{M}_{\mathcal{B}}^{\varrho}}(\diamond(\neg H \vee \neg E)) .
$$

We can then derive:

$$
\begin{aligned}
& \operatorname{Pr}(H \mid E) \\
&= \frac{\operatorname{Pr}_{\mathcal{B}}(H \wedge E)}{\operatorname{Pr}_{\mathcal{B}}(E)} \\
& \text { 10] } \frac{\operatorname{Pr}_{\mathcal{M}_{\mathcal{B}}^{o}}\left(\square\left(H^{*} \wedge E^{*}\right)\right)}{\operatorname{Pr}_{\mathcal{M}_{\mathcal{B}}^{o}}\left(\square E^{*}\right)} \\
& \stackrel{\text { and 12 }}{=} \frac{1-\operatorname{Pr}_{\mathcal{M}_{\mathcal{B}}^{o}}(\diamond(\neg H \vee \neg E))}{1-\operatorname{Pr}_{\mathcal{M}_{\mathcal{B}}^{o}}(\diamond \neg E)} .
\end{aligned}
$$

This finalizes the proof for Proposition 1

Formalizing the operations. We now proceed towards the proof of Proposition 2 by first formalizing the two operations to construct the evidence-tailored MC of $\mathrm{BN}$ : propagation and redirection.

Definition 4 (Propagation). Let $\mathcal{M}_{\mathcal{B}}^{\varrho}$ be the evidence-agnostic $M C$ of $B N \mathcal{B}$ for the topological order $\varrho$. Applying the propagation operation on $\mathcal{M}_{\mathcal{B}}^{\varrho}$ yields the $M C \mathcal{M}_{\mathcal{B}}^{\varrho \downarrow}=\left(S^{\downarrow}, s_{0}^{\downarrow}, P^{\downarrow}\right)$, where

$-s_{0}^{\downarrow}=s_{0}$ is the initial state.

- $S^{\downarrow}=\bigcup_{i=0}^{m} S_{i}^{\downarrow}$ is the set of states defined as follows:

- For $i=0$ and $i>\varrho\left(v_{E_{\text {last }}}\right)$, $S_{i}^{\downarrow}=S_{i}$, where $S_{i}$ is the set of states at level $i$ of $\mathcal{M}_{\mathcal{B}}^{\varrho}$.

- For $0<i \leq \varrho_{E_{\text {last }}}$, $S_{i}^{\downarrow}=\{\underbrace{s_{i-1}^{\downarrow}\left[v_{i}=d_{i}\right]}_{\text {short } s^{\downarrow}} \mid$ for $s_{i-1}^{\downarrow} \in S_{i-1}^{\downarrow}$ and $d_{i} \in D_{v_{i}}\}$, with

- $s^{\downarrow}\left(v_{i}\right)=d_{i}$,

- $s^{\downarrow}(v)=s_{i-1}^{\downarrow}(v)$ for $v \neq v_{i}$ iff either (I) $\varrho(v)<\varrho\left(v_{i}\right)$ and $\exists c \in$ children $(v) . \varrho(c)>\varrho\left(v_{i}\right)$ or (II) $v \notin \operatorname{vars}(E)$,

- and $s^{\downarrow}(v)=*$, otherwise.

- $P^{\downarrow}$ is defined analogously to $P$. 
The MC $\mathcal{M}_{\mathcal{B}}^{\varrho \downarrow}$ differs from $\mathcal{M}_{\mathcal{B}}^{\varrho}$ for $0<i \leq \varrho\left(v_{E_{\text {last }}}\right)$ as imposed by constraint (II).

Lemma 2. The propagation operation ensures that

$$
\operatorname{Pr}_{\mathcal{B}}(H \mid E)=\frac{\operatorname{Pr}_{\mathcal{M}_{\mathcal{B}}^{\varrho \downarrow}}\left(\square\left(H^{*} \wedge E^{*}\right)\right)}{\operatorname{Pr}_{\mathcal{M}_{\mathcal{B}}^{\varrho \downarrow}}\left(\square E^{*}\right)} .
$$

Proof ${ }^{14}$. Definition 4 ensures a strong correspondence between the paths in $\mathcal{M}_{\mathcal{B}}^{\varrho}$ and $\mathcal{M}_{\mathcal{B}}^{\varrho \downarrow}: \pi^{\downarrow}=s_{0}^{\downarrow} s_{1}^{\downarrow} \ldots \in \operatorname{Paths}\left(\mathcal{M}_{\mathcal{B}}^{\varrho \downarrow}, \diamond E\right)$ if and only if there is the unique path $\pi=s_{0} s_{1} \ldots \in \operatorname{Paths}\left(\mathcal{M}_{\mathcal{B}}^{\varrho}, \square E^{*}\right)$ such that for each $i \in \mathbb{N}$,

$$
P^{\downarrow}\left(s_{i}^{\downarrow}, s_{i+1}^{\downarrow}\right)=P\left(s_{i}, s_{i+1}\right) .
$$

It follows that

$$
\operatorname{Pr}_{\mathcal{M}_{\mathcal{B}}^{\varrho}}\left(\square E^{*}\right)=\operatorname{Pr}_{\mathcal{M}_{\mathcal{B}}^{\rho \downarrow}}\left(\square E^{*}\right)
$$

and analogously for $H \wedge E$,

$$
\operatorname{Pr}_{\mathcal{M}_{\mathcal{B}}^{\varrho}}\left(\square\left(H^{*} \wedge E^{*}\right)\right)=\operatorname{Pr}_{\mathcal{M}_{\mathcal{B}}^{\varrho \downarrow}}\left(\square\left(H^{*} \wedge E^{*}\right)\right) .
$$

Equation (13) derives from the equations (14) and (15), and Proposition $1 \quad \nabla$

We now proceed by formalizing the redirection operation for an arbitrary MC. Let $\mathcal{M}=\left(S, s_{0}, P\right)$ be a $\mathrm{MC}$ and $A P=\left\{a_{i} \mid i \in \mathbb{N}\right\}$ be a set of atomic propositions. Let $L: S \rightarrow 2^{A P}$ be a labelling function that maps each state of $\mathcal{M}$ to a finite subset of atomic propositions. Let $\phi=\bigwedge_{i=1}^{k} a_{i}$ and $S_{\neg \phi}$ denote the set of states that violate $\phi$, i.e.,

$$
S_{\neg \phi}=\left\{s \in S \mid s \models \neg a_{i} \text { for some } 1 \leq i \leq k\right\} .
$$

The redirection operation reroutes the direct transitions to the states in $S_{\neg \phi}$ to the initial state $s_{0}$ and deletes the states in $S_{\neg \phi}$.

Definition 5 (Redirection). Applying the redirection operation to the $M C \mathcal{M}$ with respect to $\phi$ yields the $M C(\mathcal{M})_{\phi}=\left(S \backslash S_{\neg \phi}, s_{0}, P_{\phi}\right)$, where for $s \in S \backslash S_{\neg \phi}$

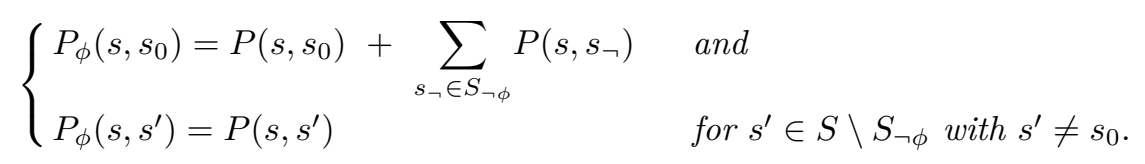

Let $G$ be a set of target states in $(\mathcal{M})_{\phi}$. Recall that variable $p_{s}$ denotes the probability to reach $G$ from state $s$. Let $\operatorname{succ}(s)$ denote the set of direct successors

$\overline{14}$ The proof is analogous to Lemma 1 . 


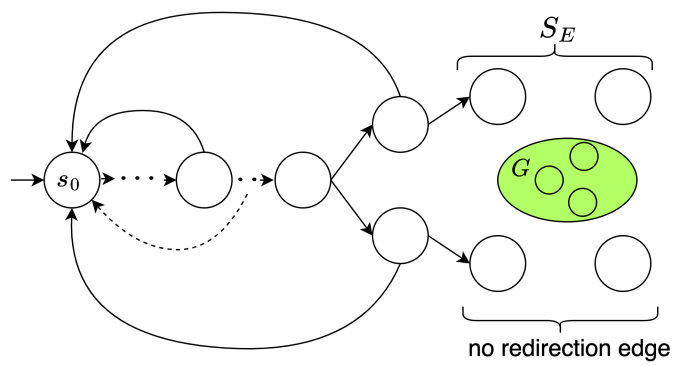

Fig. 7: The goal states $G$ occur after the last redirection loop of $\mathcal{M}_{\mathcal{B}, E}: G \subseteq S_{E}$.

of $s$ in the MC $\mathcal{M}$. It follows by equation (5) and Def. 5 that for the state $s \in S \backslash S_{\neg \phi}$,

$$
p_{s}=\sum_{s^{\prime} \in \operatorname{succ}(s) \backslash S_{\neg \phi}} P\left(s, s^{\prime}\right) \cdot p_{s^{\prime}}+\underbrace{\sum_{s_{\neg} \in \operatorname{succ}(s) \cap S_{\neg \phi}} P\left(s, s_{\neg}\right) \cdot p_{s_{0}}}_{\text {redirection to the initial state }} .
$$

Query correspondence for the evidence-tailored $M C$. The evidence-tailored MC $\mathcal{M}_{\mathcal{B}, E}^{\varrho}=\left(S^{\downarrow} \backslash S_{\neg E}^{\downarrow}, s_{0}^{\downarrow}, P_{E}\right)$ is obtained by applying the redirection operation to the $\mathrm{MC} \mathcal{M}_{\mathcal{B}}^{\varrho \downarrow}$ with respect to $\phi=E$, i.e., $\mathcal{M}_{\mathcal{B}, E}^{\varrho}=\left(\mathcal{M}_{\mathcal{B}}^{\varrho \downarrow}\right)_{E}$. Let $S_{E}=$ $\bigcup_{i=\varrho\left(v_{E_{\text {last }}}\right)}^{m} S_{i}^{\downarrow}$ be the set of states in $\mathcal{M}_{\mathcal{B}, E}^{\varrho}$ that only occurs at the level of the last evidence node and afterwards, i.e., the set of states that occur after the last redirection edge, see Fig. 7 .

Lemma 3. For $G \subseteq S_{E}$, it holds that

$$
\operatorname{Pr}_{\mathcal{B}, E}^{\rho}(\diamond G)=\frac{\operatorname{Pr}_{\mathcal{M}_{\mathcal{B}}^{\varrho \downarrow}}\left(\diamond G \wedge \square E^{*}\right)}{\operatorname{Pr}_{\mathcal{M}_{\mathcal{B}}^{\rho \downarrow}}\left(\square E^{*}\right)} .
$$

Proof. We prove the above lemma by rewriting equation 16 for $\mathcal{M}_{B, E}^{\varrho}$. First, let $G \subseteq S^{\downarrow}$ be arbitrary. Recall that $S_{i}^{\downarrow}$ denotes the set of states at the $i$-th level of $\mathcal{M}_{\mathcal{B}}^{\varrho \downarrow}$

- For each $s_{i}^{\downarrow} \in S_{i}^{\downarrow} \cap \operatorname{pre} e^{*}(G)$,

$$
p_{s_{i}^{\downarrow}}=\sum_{s_{i+1}^{\downarrow} \in S_{i+1}^{\downarrow} \backslash S_{\neg E}^{\downarrow}} P^{\downarrow}\left(s_{i}^{\downarrow}, s_{i+1}^{\downarrow}\right) \cdot p_{s_{i+1}^{\downarrow}}+\sum_{t_{i+1}^{\downarrow} \in S_{i+1}^{\downarrow} \cap S_{\neg E}^{\downarrow}} P^{\downarrow}\left(s_{i}^{\downarrow}, t_{i+1}^{\downarrow}\right) \cdot p_{s_{0}^{\downarrow}},
$$

- for each $s_{i}^{\downarrow} \notin \operatorname{pre}^{*}(G), p_{s_{i}^{\downarrow}}=0$,

\footnotetext{
$\overline{15}$ Note that by Def. 4 for every $s_{i}^{\downarrow} \in S_{i}^{\downarrow}, \operatorname{succ}\left(s_{i}^{\downarrow}\right) \subseteq S_{i+1}^{\downarrow}$.
} 
- and for each $s_{i}^{\downarrow} \in G, p_{s_{i}^{\downarrow}}=1$.

Let $\neg E_{1 \ldots j}=\bigcup_{i=1}^{\varrho\left(v_{E_{j}}\right)} S_{i}^{\downarrow} \cap S_{\neg E}^{\downarrow}$ specify the states that violate $E_{1}$ up to $E_{j}$. The above equation system yields the following.

For $G \subseteq \bigcup_{i=\varrho\left(v_{E_{j}}\right)}^{m} S_{i}^{\downarrow}$

$$
p_{s_{0}^{\downarrow}}=\sum_{\pi \in \operatorname{Paths}\left(\mathcal{M}_{\mathcal{B}}^{\varrho \downarrow}, \diamond G\right) \backslash \operatorname{Paths}\left(\mathcal{M}_{\mathcal{B}}^{\varrho \downarrow}, \diamond \neg E_{1 \ldots j}\right)} \operatorname{Pr}(\pi) \quad p_{\substack{s_{0}^{\downarrow} \\ \pi^{\prime} \in \operatorname{Paths}\left(\mathcal{M}_{\mathcal{B}}^{\varrho \downarrow}, \diamond \neg E_{1 \ldots j}\right)}} \operatorname{Pr}\left(\pi^{\prime}\right)
$$

and for $G \subseteq \bigcup_{i=\varrho\left(v_{E_{\text {last }}}\right)}^{m} S_{i}^{\downarrow}$,

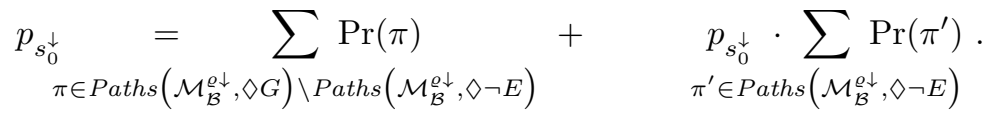

We have $\neg(\diamond \neg E) \equiv \square E^{*}$ (Equations (6) and (11)). Moreover, note that $A \backslash B=$ $A \cap \neg B$. We can thus rewrite equation (17) as

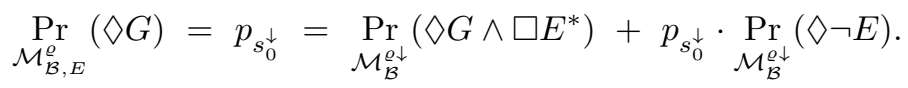

Thus:

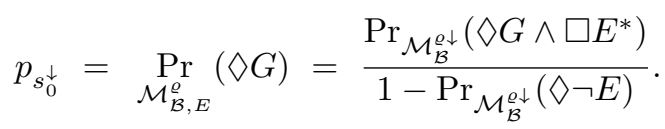

Note that $1-\operatorname{Pr}_{\mathcal{M}_{\mathcal{B}}^{\varrho \downarrow}}(\diamond \neg E)=\operatorname{Pr}_{\mathcal{M}_{\mathcal{B}}^{\varrho \downarrow}}\left(\square E^{*}\right)$. This ends the proof.

Proposition 2. For the evidence-tailored $\mathrm{pMC} \mathcal{M}_{\mathcal{B}, E}^{\varrho}$ of $\mathrm{pBN} \mathcal{B}$, we have:

$$
\operatorname{Pr}_{\mathcal{B}}(H \mid E)=1-\operatorname{Pr}_{\mathcal{M}_{\mathcal{B}, E}^{\varrho}}\left(\diamond\left(\left(\neg H_{\text {before }} \wedge E_{\text {last }}\right) \vee \neg H_{\text {after }}\right)\right) .
$$

Proof. We apply Lemma 3 to the evidence-tailored $\mathrm{MC} \mathcal{M}_{\mathcal{B}, E}^{\varrho}$. Let $G=$ $\left.\left(\left(\neg H_{\text {before }} \wedge E_{\text {last }}\right) \vee \neg H_{\text {after }}\right)\right)$. Recall that $S_{E}$ is the set of states at the levels $i$ with $i \geq \varrho\left(v_{E_{\text {last }}}\right)$. We have $G \subseteq S_{E}$ : the term $\neg H_{\text {before }} \wedge E_{\text {last }}$ can first be satisfied at level $i$ with $i \geq \varrho\left(v_{E_{\text {last }}}\right)$. The term $H_{\text {after }}$ can also first be satisfied at levels $i$ with $i>\varrho\left(v_{E_{\text {last }}}\right)$ by the definition of $H_{\text {after }}$.

It then follows by Lemma 3 that

$$
\begin{aligned}
& 1-\operatorname{Pr}_{\mathcal{M}_{\mathcal{B}, E}^{\varrho}}(\diamond G) \\
= & \frac{\operatorname{Pr}_{\mathcal{M}_{\mathcal{B}}^{\varrho \downarrow}}\left(\square E^{*}\right)-\operatorname{Pr}_{\mathcal{M}_{\mathcal{B}}^{\varrho \downarrow}}\left(\diamond G \wedge \square E^{*}\right)}{\operatorname{Pr}_{\mathcal{M}_{\mathcal{B}}^{\varrho \downarrow}}\left(\square E^{*}\right)}
\end{aligned}
$$




$$
\begin{aligned}
& \operatorname{Pr}(a)-\operatorname{Pr}(a \wedge b)=\operatorname{Pr}(a \wedge \neg b) \frac{\operatorname{Pr}_{\mathcal{M}_{\mathcal{B}}^{\varrho \downarrow}}\left(\square E^{*} \wedge \neg \downarrow G\right)}{\operatorname{Pr}_{\mathcal{M}_{\mathcal{B}}^{\varrho \downarrow}}\left(\square E^{*}\right)} \\
& \neg \diamond a \equiv \neg \neg a \frac{\operatorname{Pr}_{\mathcal{M}_{\mathcal{B}}^{\varrho \downarrow}}\left(\square E^{*} \wedge \square \neg G\right)}{\operatorname{Pr}_{\mathcal{M}_{\mathcal{B}}^{\varrho \downarrow}}\left(\square E^{*}\right)} \\
& \square \neg(a \vee b) \equiv \square \neg a \wedge \square \neg b \frac{\operatorname{Pr}_{\mathcal{M}_{\mathcal{B}}^{\varrho \downarrow}}\left(\square E^{*} \wedge \square \neg\left(\neg H_{\text {before }} \wedge E_{\text {last }}\right) \wedge \neg\left(\neg H_{\text {after }}\right)\right)}{\operatorname{Pr}_{\mathcal{M}_{\mathcal{B}}^{\varrho \downarrow}}\left(\square E^{*}\right)}
\end{aligned}
$$

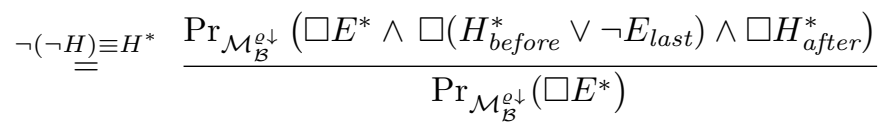

$$
\begin{aligned}
& \square a \wedge \square b \equiv \wedge \square(a \wedge b) \frac{\operatorname{Pr}_{\mathcal{M}_{\mathcal{B}}^{\varrho \downarrow}}\left(\square\left(E^{*} \wedge\left(H_{\text {before }}^{*} \vee \neg E_{\text {last }}\right)\right) \wedge \square H_{\text {after }}^{*}\right)}{\operatorname{Pr}_{\mathcal{M}_{\mathcal{B}}^{\varrho\llcorner}}\left(\square E^{*}\right)} \\
& =\frac{\operatorname{Pr}_{\mathcal{M}_{\mathcal{B}}^{\rho \downarrow}}\left(\square\left(\left(E^{*} \wedge H_{\text {before }}^{*}\right) \vee\left(E^{*} \wedge \neg E_{\text {last }}\right)\right) \wedge \square H_{\text {after }}^{*}\right)}{\operatorname{Pr}_{\mathcal{M}_{\mathcal{B}}^{\rho \downarrow}}\left(\square E^{*}\right)}
\end{aligned}
$$

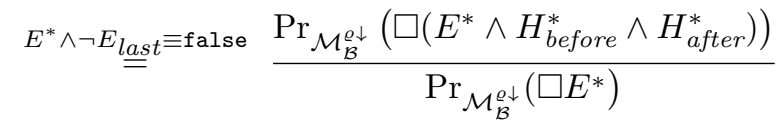

$$
\begin{aligned}
& H_{\text {before }}^{*} \stackrel{\wedge H_{\text {after }}^{*} \equiv H^{*}}{=} \frac{\operatorname{Pr}_{\mathcal{M}_{\mathcal{B}}^{\varrho \downarrow}}\left(\square\left(E^{*} \wedge H^{*}\right)\right)}{\operatorname{Pr}_{\mathcal{M}_{\mathcal{B}}^{\varrho \downarrow}}\left(\square E^{*}\right)} .
\end{aligned}
$$

It then follows by Lemma 2 that

$$
1-\operatorname{Pr}_{\mathcal{M}_{\mathcal{B}, E}^{o}}\left(\diamond\left(\left(\neg H_{\text {before }} \wedge E_{\text {last }}\right) \vee \neg H_{\text {after }}\right)\right)=\operatorname{Pr}_{\mathcal{B}}(H \mid E),
$$

which finalizes the proof for Proposition 2.

\section{B Some detailed experimental results}

Figure 8 and Tables 2 , 3, and 4 provide a detailed overview on some of our experiments for $\mathrm{pBN}$ parameter tuning.

Storm's precision for minimal change parameter tuning. Figure 8 provides an overview of how precise the results of parameter space partitioning experiments are in comparison to Bayesserver, one of our baseline tools. Note that Bayesserver reports the result with higher precision in comparison to SamIam. We took a $p_{1} c_{1} d_{1}$ subclass from alarm network and performed approximate parameter tuning using our tool. Each point indicates a single experiment. The plot is 
in $\log$-log scale. The $\mathrm{x}$-axis indicates the approximation factor $(1-c)$ for the experiments; the y-axis indicates how much the results - the minimal distance point - found by PLA deviates from the results reported by Bayesserver. We see that the precision tightly depends on the approximation factor and as we increase PLA's coverage - i.e., smaller approximation factor-, the results get closer to that of reported by Bayesserver. The results coincide at $1-c=10^{-16}$.

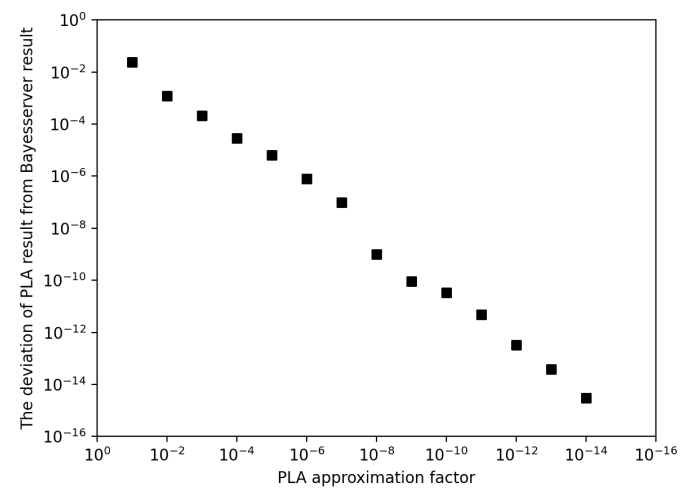

Fig. 8: The difference between Storm results and Bayesserver results for various PLA approximation factors, $p_{1} c_{1} d_{1}$ subclass, alarm network.

Appendix tables description. Table 2 (page 27) includes the detailed results for computing sensitivity function. For each network, three pBN versions were considered: $p_{1} c_{1} r_{1}, p_{2} c_{\leq} r_{1}$, and $p_{*} c_{*} r_{1}$. Column "\#parameters" indicates the total number of parameters. Column "Storm (regular)" indicates the model checking time taken by Storm for computing the sensitivity functions, while the column "Storm (bi-simulation)" indicates the sensitivity function computation time when exploiting bisimulation minimization. The last column indicates the corresponding sensitivity analysis time for Bayesserver, for the same $\mathrm{pBN}$ and query. Note that Bayesserver does not support the class $p_{*} c_{*} r_{1}$, thus we compared the results for the subclasses $p_{1} c_{1} r_{1}$ and $p_{2} c_{\leq} r_{1}$. We see that Storm (when using the bi-simulation engine) outperforms Bayesserver in most of the experiments.

Table 3 (page 28) indicates our experimental results for parameter tuning on the $p_{1} c_{1} d_{1}$ class, the only subclass supported by both baseline tools. For Storm, the column "synthesis time" indicates the time taken by PLA to tune the parameters. "Total CPU time" indicates the total time including model building and parsing time. The "parameter space coverage" is related to the approximation factor taken for PLA; it indicates the percentage of the entire parameter space covered by PLA for the synthesis. The column "threshold found" indicates the safe interval found by PLA for the given constraint. For Bayesserver and SamIam, the columns "threshold reported" indicate the interval reported for the same pBN and constraint. The table also includes the "tuning time" and the "total time" for Bayesserver, which respectively are the time for the 
parameter tuning process and the total time -including the time for building and parsing the model. The synthesis time for Bayesserver is often less than Storm. This is because Bayesserver uses the existing closed-form formula to find the parameter instantiation for this specific subclass $\left(p_{1} c_{1} d_{1}\right)$, while Storm does not exploit closed-form formulas and model-checks the entire parameter space. It determines the accepting and rejecting areas and is in principle applicable to arbitrary subclass $p_{*} c_{*} d_{*}$, see Tab. 4. For two of the benchmarks Storm encountered time-out and memout while constructing the MC.

Tab. 4 (page 29 indicates the PLA experiments performed on the alarm BN benchmark. The column "\#parameterised rows" indicates the number of CPT rows parameterized in the $\mathrm{pBN}$, followed by the pBN class the benchmark belongs to. The subclass specifies the number of parameters, as well as the number of parametrized CPTs and the number of CPT rows the parameters are repeated at. The latter is related to the non-distinct parameters. The third column indicates the refinement factor taken for PLA. The fourth column indicates the number of regions synthesized by PLA; followed by the percentage of satisfied and unknown regions. The last four columns indicate the time taken by PLA for synthesis, the time taken by Storm for model building, the total process time, and the memory footprint. MO and TO respectively abbreviates "memory-out" and "time-out" of 15 minutes in tables 3 and 4 . 
Table 2: Solution function calculation for the subclasses $p_{1} c_{1} d_{1}, p_{2}, c_{\leq 2} d_{1}$, and $p B N$ - Storm vs Bayesserver

\begin{tabular}{|c|c|c|c|c|}
\hline & |\# parameters & Storm (regular) & $\mid$ Storm (bi-simulation) $\mid$ & Bayesserver \\
\hline \multirow[t]{3}{*}{ cancer } & 1 (one way) & $0.000 \mathrm{~s}$ & $0.000 \mathrm{~s}$ & $0.011 \mathrm{~s}$ \\
\hline & 2 (two way) & $0.000 \mathrm{~s}$ & $0.000 \mathrm{~s}$ & $0.010 \mathrm{~s}$ \\
\hline & 10 & $0.000 \mathrm{~s}$ & $0.000 \mathrm{~s}$ & not supported \\
\hline \multirow[t]{3}{*}{ earthquake } & 1 (one way) & $0.000 \mathrm{~s}$ & $0.000 \mathrm{~s}$ & $0.011 \mathrm{~s}$ \\
\hline & 2 (two way) & $0.000 \mathrm{~s}$ & $0.000 \mathrm{~s}$ & $0.011 \mathrm{~s}$ \\
\hline & 10 & $0.000 \mathrm{~s}$ & $0.000 \mathrm{~s}$ & not supported \\
\hline \multirow[t]{3}{*}{ asia } & 1 (one way) & $0.001 s$ & $0.003 \mathrm{~s}$ & $0.0164 \mathrm{~s}$ \\
\hline & 2 (two way) & $0.000 \mathrm{~s}$ & $0.000 \mathrm{~s}$ & $0.0168 \mathrm{~s}$ \\
\hline & 14 & $0.009 \mathrm{~s}$ & $0.000 \mathrm{~s}$ & not supported \\
\hline \multirow[t]{3}{*}{ sachs } & 1 (one way) & $0.007 \mathrm{~s}$ & $0.002 \mathrm{~s}$ & $0.0100 \mathrm{~s}$ \\
\hline & 2 (two way) & $0.000 \mathrm{~s}$ & $0.000 \mathrm{~s}$ & $0.0103 \mathrm{~s}$ \\
\hline & 89 & $1.878 \mathrm{~s}$ & $1.582 \mathrm{~s}$ & not supported \\
\hline \multirow[t]{3}{*}{ alarm } & 1 (one way) & $0.011 \mathrm{~s}$ & $0.002 \mathrm{~s}$ & $0.018 \mathrm{~s}$ \\
\hline & 2 (two way) & $0.025 \mathrm{~s}$ & $0.004 \mathrm{~s}$ & $0.027 \mathrm{~s}$ \\
\hline & 85 & $872 \mathrm{~s}$ & 201s & not supported \\
\hline \multirow[t]{3}{*}{ insurance } & 1 (one way) & $0.199 \mathrm{~s}$ & $0.038 \mathrm{~s}$ & $0.011 s$ \\
\hline & 2 (two way) & 0.178 & $0.036 \mathrm{~s}$ & $0.034 \mathrm{~s}$ \\
\hline & 140 & $259 \mathrm{~s}$ & $83 \mathrm{~s}$ & not supported \\
\hline \multirow[t]{3}{*}{ win95pts } & 1 (one way) & $0.002 \mathrm{~s}$ & $0.000 \mathrm{~s}$ & $0.0186 \mathrm{~s}$ \\
\hline & 2 (two way) & $0.002 \mathrm{~s}$ & $0.000 \mathrm{~s}$ & $0.0148 \mathrm{~s}$ \\
\hline & 200 & $548 \mathrm{~s}$ & $437 \mathrm{~s}$ & not supported \\
\hline \multirow[t]{3}{*}{ hepar2 } & 1 (one way) & $0.148 \mathrm{~s}$ & $0.033 \mathrm{~s}$ & $0.020 \mathrm{~s}$ \\
\hline & 2 (two way) & $0.130 \mathrm{~s}$ & $0.026 \mathrm{~s}$ & $0.020 s$ \\
\hline & 135 & $193 \mathrm{~s}$ & $172 \mathrm{~s}$ & not supported \\
\hline \multirow[t]{3}{*}{ hailfinder } & 1 (one way) & $0.077 \mathrm{~s}$ & $0.000 \mathrm{~s}$ & $0.009 \mathrm{~s}$ \\
\hline & 2 (two way) & $0.080 \mathrm{~s}$ & $0.000 \mathrm{~s}$ & $0.009 \mathrm{~s}$ \\
\hline & 380 & $38 \mathrm{~s}$ & $33 \mathrm{~s}$ & not supported \\
\hline \multirow[t]{3}{*}{ water } & 1 (one way) & $0.081 \mathrm{~s}$ & $0.017 \mathrm{~s}$ & $0.058 \mathrm{~s}$ \\
\hline & 2 (two way) & $0.089 \mathrm{~s}$ & $0.012 \mathrm{~s}$ & $0.107 \mathrm{~s}$ \\
\hline & 255 & $246 s$ & $247 \mathrm{~s}$ & not supported \\
\hline
\end{tabular}




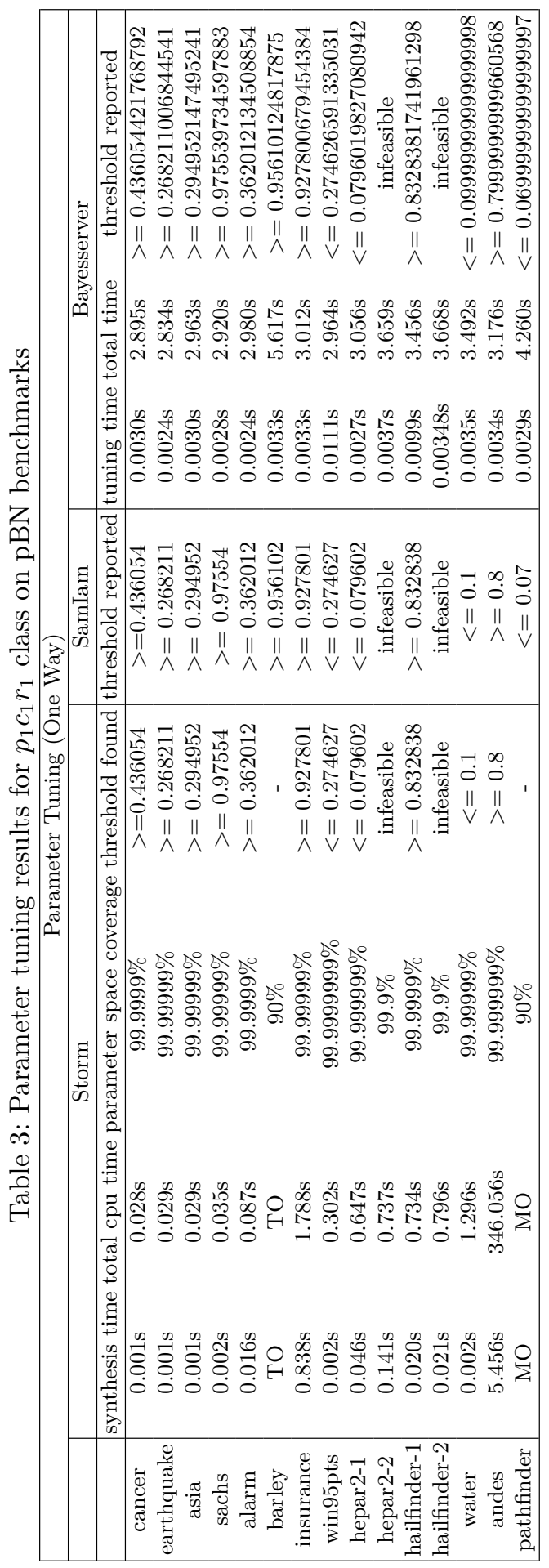




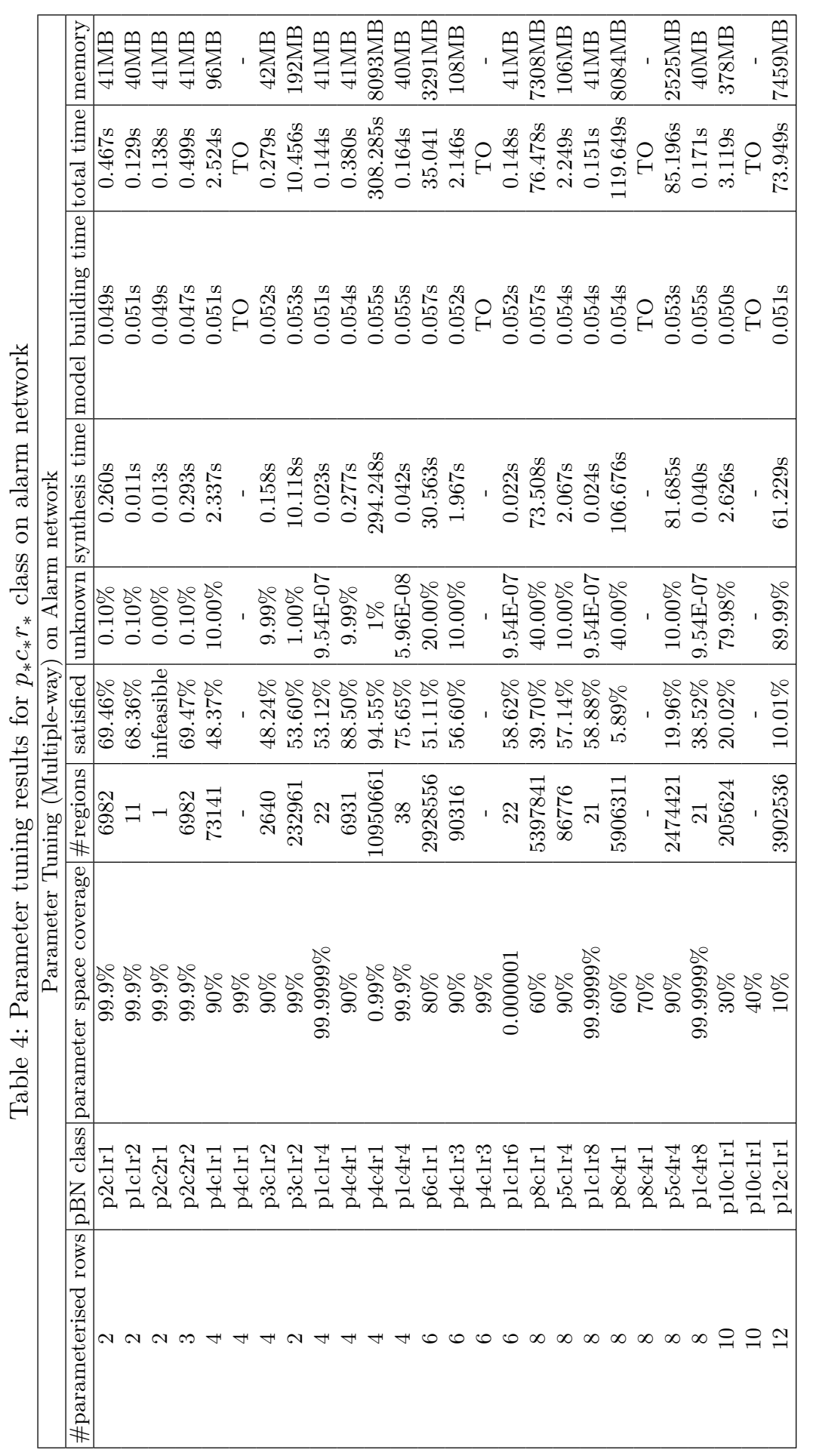

